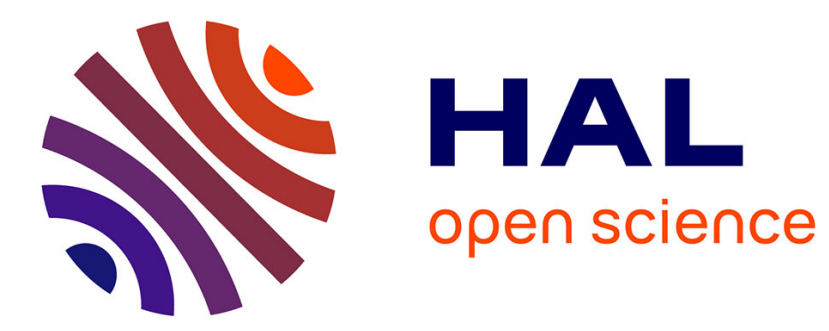

\title{
Each Other, Asymmetry and Reasonable Futures
}

Alda Mari

\section{To cite this version:}

Alda Mari. Each Other, Asymmetry and Reasonable Futures. Journal of Semantics, 2014, 31 (2), pp.209 - 261. 10.1093/jos/fft003 . ijn_02161864

\section{HAL Id: ijn_02161864 \\ https://hal.science/ijn_02161864}

Submitted on 21 Jun 2019

HAL is a multi-disciplinary open access archive for the deposit and dissemination of scientific research documents, whether they are published or not. The documents may come from teaching and research institutions in France or abroad, or from public or private research centers.
L'archive ouverte pluridisciplinaire HAL, est destinée au dépôt et à la diffusion de documents scientifiques de niveau recherche, publiés ou non, émanant des établissements d'enseignement et de recherche français ou étrangers, des laboratoires publics ou privés. 


\title{
Each other, asymmetry and reasonable futures
}

\author{
Alda Mari \\ November 28, 2012 \\ Journal of Semantics, Volume 31, Issue 2, May 2014, Pages 209-261 \\ To appear, Journal of Semanties. Pre-final version. \\ Please, see published paper for precise content and citation
}

\begin{abstract}
Reciprocal sentences display a variety of interpretations, including 'strong reciprocity'. In this interpretation, every element of the reference set participates with every other element in the same set in the relation provided by the predicate. Another interpretation is 'inclusive alternative orderings'. In this interpretation, every element in the reference set participates with some other member in the relation provided by the predicate either as the first or second argument. Current reciprocal theories cannot explain why some sentences that satisfy these truth conditions are in fact false and infelicitous, such as '\#my mother and I gave birth to each other'.

This paper defends the view that strong reciprocity has a privileged status. Its core insight is that reciprocal sentences are true if they describe a relation that is either actually or possibly strong reciprocal over the reference set, insofar as the possibilities are reasonable. The new truth conditions are cast in a branching time framework (Thomason, 1984), in which a new notion of reasonability is defined (vs. inertia, Dowty, 1979; see also Landman, 1992).

On the empirical side, we show that when the relation is asymmetric, it must be non-permanent for the reciprocal each other-sentence to be true. We also note that, in some cases, the relation is asymmetric and non-permanent, but the each other-sentences are false. We explain these facts by introducing a new notion of 'decidedness' that we define in modal terms. We explain that the relation should not be decided for the reciprocal sentence to be true. We also provide a new definition for asymmetry and (non-)permanency in the modal framework and explain how asymmetry, (non-)decidedness and (non-)permanency interact, ensuring large empirical coverage.
\end{abstract}

Keywords: Reciprocity, asymmetry, decidedness, permanency, branching time, reasonable worlds, statives. 


\section{Introduction}

The semantic variability of reciprocal each other-sentences is a well-known phenomenon. ${ }^{1}$ Among the available interpretations of such sentences, there is 'strong reciprocity'. In this interpretation, a sentence such as (1) is interpreted as 'each man in the set of men knows every other man but himself in the set of men'.

(1) The men know each other.

Another available interpretation is 'inclusive alternative orderings'. In this interpretation, a sentence such as (2) is interpreted as 'for each table $x$ in the set of tables, there is a different one $y$ in the same set, such that either $x$ is on top of $y$ or $y$ is on top of $x^{\prime} .^{2}$

(2) The tables are stacked on top of each other.

Foundational accounts held that reciprocal each other-sentences only primarily had a strong reciprocal interpretation (e.g., Dougherty, 1968; Heim, Lasnik and May, 1991). In the flourishing subsequent literature, observing that reciprocal each other-sentences display a variety of interpretations, theoreticians have abandoned this idea and have assigned a variety of truth conditions to such statements. ${ }^{3}$ Let $A$ stand for a plural (i.e., non-singleton) set and $R$ for a binary relation over $A \times A$. Sentence (1) satisfies the truth conditions in (3). Sentence (2) is assigned the truth conditions in (4). In both (3) and (4), the condition $x \neq y$ guarantees that the relation denotes pairs of distinct individuals in the same set. The truth of the reciprocal sentence does not depend on whether each member of the reference set is in relation $R$ with himself. Heim, Lasnik and May (1992) labeled this condition as 'diversity condition'. $\langle x, y\rangle$ is the ordered pair with $x$ as first component and $y$ as second component.

$\forall x \in A \forall y \in A(x \neq y \rightarrow R\langle x, y\rangle)$

Paraphrase: for every $x$ in the set $A$, for every $y$ in the set $A$, if $x$ is different from $y$, then $x$ stands in the relation $R$ with $y$.

$\forall x \in A \exists y \in A(x \neq y \wedge(R\langle x, y\rangle \vee R\langle y, x\rangle))$

Paraphrase: for every $x$ in the set $A$, there is a $y$ in the set $A, x$ is different from $y$ and either $x$ stands in the relation $R$ with $y$ or $y$ stands in the relation $R$ with $x$.

This paper acknowledges the variety of available interpretations but defends the idea that strong reciprocity has a privileged role in the semantics of reciprocal each other-sentences.

\footnotetext{
${ }^{1}$ A.o. Fiengo and Lasnik, 1973; Langendoen, 1978, Dalrymple et al. 1998; Beck, 2001, Kerem, Friedmann and Winter, 2009; Sabato and Winter, 2005,2012.

${ }^{2}$ Fiengo and Lasnik (1973) first identified this meaning. See also a.o. Langendoen, 1978; Schwarzschild, 1996; Dalrymple et al. 1998; Beck, 2001.

${ }^{3}$ See, e.g., Langendoen, 1978; Dalrymple et al. 1998; Beck, 2001.
} 
Its core insight is that reciprocal sentences are true if they describe a relation that is either actually or possibly strong reciprocal over the reference set, insofar as the possibilities are reasonable. We will thus abandon (3) and (4) and do not retain them any longer as truth conditions for sentences (1) and (2). The new truth conditions are cast in a branching time framework (Thomason, 1984), in which the notion of 'reasonability' is defined in terms of normality conditions (Dowty, 1979; Landman, 1992).

This paper is mainly concerned with sentences such as (2), as the truth conditions that have been assigned to these sentences, namely (4), remain challenging for all theories of reciprocals of which we are aware.

Let us briefly explain what the challenges are.

There are essentially two accounts for the variety of the interpretations of reciprocal each other-sentences.

One view, which has become popular since the work of Heim, Lasnik and May (1991), holds that each other is a pronominal DP, meaning 'the others among them' (Beck, 2001:69). In this framework, Beck (ibid.) has argued that the source of the variety of the interpretations is the indeterminacy that one finds in relational plural sentences. (5) is a relational plural sentence.

(5) The three children know the two books.

In (5), there are two expressions denoting two plural (i.e., non-singleton) sets (i.e., the three children and two books). The predicate knows denotes a relation between these two sets. Let $A$ be the set of children, $B$ the set of books and $R$ the relation know. A relational plural sentence such as (5) has an interpretation in which both plural sets are universally quantified over (6).

$$
\begin{aligned}
& \forall x \in A \forall y \in B(R\langle x, y\rangle) \\
& \text { Paraphrase: Every child in the group of children knows every book in the group } \\
& \text { of books. }
\end{aligned}
$$

Consider now the reciprocal sentence (7-a) on the strong reciprocal interpretation (3) repeated in (7-b). Comparing the truth conditions in (7-b) with those in (6), the only difference is that the set $A$ is quantified twice and appropriate distinctness conditions are provided (see (7-b)).

(7) a. The three children know each other.

b. $\forall x \in A \forall y \in A(x \neq y \rightarrow R\langle x, y\rangle)$

Paraphrase: every child in the group of children knows every other child with the possible exception of himself. 
The demonstration that available interpretations of reciprocal sentences mirror available interpretations of relational plural sentences is reiterated for other available meanings of reciprocal sentences. As Beck (ibid.) concludes, the truth conditions in (4) are a challenge to this view of reciprocity, as there is no available interpretation of relational plural sentences that mirrors the truth conditions in (4) for reciprocal sentences.

Moreover, as the contrast in (8) illustrates, if the truth conditions in (4) for reciprocal sentences mirrored available interpretations of relational plural sentences, some restrictions that hold for reciprocal sentences would be expected to hold for plural sentences as well, contrary to fact. (We discuss these restrictions extensively in section 2.)
a. \#The boys are taller than each other.
b. The boys are taller than the girls.

Following Beck (2001), Beck and von Stechow (2007) propose that reciprocal sentences such as (2) should be treated separately from reciprocal sentences that mirror the readings of relational plural sentences. Beck and von Stechow abandon the idea that (2) should be assigned the truth conditions in (4) and propose new truth conditions, on which we comment below.

A second view on the reciprocal expression each other has held that each other is a quantifier and the variety of interpretations of reciprocal sentences is due to the ambiguity of the quantifier itself. This view goes back to Dalrymple et al. (1998).

Consider a simple reciprocal sentence (9), where $A$ stands for an arbitrary plural (i.e., non-singleton) set, $R$ a binary relation on $A \times A$ and $E O$ stands for the reciprocal quantifier.

\section{(9) $\quad A R E O$}

$E O$ is a polyadic quantifier of type $\langle 1,2\rangle$ that maps a set and relation into a truth value. This quantifier is considered ambiguous between a series of quantifiers. For sentence (1), the polyadic quantifier is commonly assumed to be $S R$.

$$
S R(A, R)=1 \text { iff }(1)
$$

For sentence (2), the quantifier is assumed to be $I A O$.

$$
I A O(A, R)=1 \text { iff }(4)
$$

Maintaining that the truth conditions in (4) are to be assigned to reciprocal statements such as (2), Dalrymple et al. (ibid.) and subsequent work in their tradition face the following problem. The truth conditions in (4) over-generate, as all sentences that satisfy 
these truth conditions are predicted true, contrary to fact. ${ }^{4}$

\#My mother and I gave birth to each other. ${ }^{5}$

Determining where the breakpoint stands between sentences that satisfy the truth conditions in (4) but are false (12), and those that satisfy the truth conditions in (4) and are true (2), will put us on the track of a new temporal-modal account requiring that strong reciprocity be cumulatively enacted either actually or possibly across reasonable branches of the actual world. We treat sentences such as (2) with all other available reciprocal sentences.

In this paper, we endorse the view that each other is a polyadic quantifier (in line with Keenan, 1987; Dalrymple et al. 1998; more recently, Dotlačil and Nilsen, 2009; Sabato and Winter, 2011). This choice is guided by the recent findings obtained in Dotlačil and Nilsen (2009) showing that the polyadic quantifier approach better accounts for the behavior of each other sentences than its competitors. ${ }^{6}$ Moreover, while Beck (2001) recognizes that her view cannot be extended to cover cases such as (2) and that the only available option is to treat them separately (see Beck and von Stechow, 2007), the polyadic quantifier view does not limit the possible treatments of the variety of interpretations of reciprocal sentences (see e.g., Sabato and Winter, 2012). Here, we propose a unified theory for each other sentences, which, unlike Dalrymple et al.'s (1998) approach, does not assume that the polyadic quantifier is ambiguous.

The paper is structured as follows. In section 2, we present new data that lead us to abandon (4). In section 3, we propose new truth conditions for reciprocal each other sentences. In section 4, we spell out in our formal framework the constraints that reciprocal relations should satisfy for a reciprocal each other sentence to be true. Section 5 discusses the predictions made by our account. In section 6, we discuss Schein's (2005) and Beck and von Stechow's (2007) theories that have acknowledged problems raised by the truth conditions in (4), abandoned them and proposed new truth conditions for the reciprocal sentence (2). We show that these theories cannot cover the variety of data that we discuss in section 2. Section 7 concludes the paper.

\footnotetext{
${ }^{4}$ The puzzling status of (4) had already been noted by Fiengo and Lasnik, 1973; Langendoen, 1978; more recently by Sauerland, 1998; Beck, 2001; Winter, 2001; Sabato and Winter, 2012.

${ }^{5}$ This example is a variant from Sauerland, (1998). The original example reads '\#My mother and I procreated each other'. The problem with the original example is that '*My mother procreated me' is ungrammatical.

${ }^{6}$ Dotlačil and Nilsen (2009) show that Beck's (2001) view is more suitable for accounting for the properties of the reciprocal one another.
} 


\section{Constraints on asymmetric predicates: old and new observations}

Throughout this section, let $A$ stand for a plural (i.e., non-singleton) set and $R$ for a binary relation over $A \times A$.

\subsection{Asymmetric predicates and IAO interpretation: previous ob- servations}

Dalrymple et al. (1998) claim that the 'inclusive alternative orderings' interpretation of reciprocal sentences (see (4)) can only be obtained with asymmetric relations, on the standard assumption that relation $R$ is asymmetric on $A$ if and only if, for any $x, y \in A$ (such that $x \neq y$ ), if $x$ stands in the relation $R$ with $y$, then $y$ does not stand in the relation $R$ with $x .^{7}$

Beck (2001) reports some cases in which the relation is asymmetric and the truth conditions in (4) are satisfied, but the sentence is judged unacceptable (since it satisfies the truth conditions in (4), the sentence is expected to be true and acceptable).

The first case in point are comparatives (Higginbotham, 1981; Schwarzschild, 1996).

(13) \#John and Mary are taller than each other.

Second, Beck notes that, even if all sentences in (14) and (15) have truth conditions in (4), only those sentences in which the reference set denotes a large group are judged true and acceptable on an 'inclusive alternative orderings' interpretation. (14-b) is instead only judged true and acceptable in a scenario in which the two kings keep succeeding each other in turn. The same observation holds for (15-b).

(14) a. The kings succeeded each other.

b. (\#) The two kings succeeded each other.

a. These men inherited the shop from each other.

b. (\#) The two men inherited the shop from each other.

Third, crediting Langendoen (1978), Beck notes that temporal (16-a) and geometric properties (17-a) allow two-member pluralities. In these cases, there is no contrast between small and large groups.

(16) a. The two boys followed each other into the elevator.

b. The boys followed each other into the elevator.

\footnotetext{
${ }^{7}$ We will provide a definition of asymmetry in a temporal-modal framework in section 4 . Let us for now adopt Dalrymple et al. (ibid.) definition.
} 
a. The two tables are stacked on top of each other.

b. The tables are stacked on top of each other. $(=(2))$

Beck (ibid.) finally acknowledges the contrast between (16-a) and (12) and develops an account to which we return in section 6 . This account relies on time and events.

In distinguishing temporal from geometrical properties, it is not entirely clear what Langendoen (ibid.) and Beck (ibid.) after him intend by 'temporal' properties. If this label is used to express the idea that the property is located in time, it is not clear why spatial properties such as stacked on should be distinguished from temporal properties, as all properties are in fact located in time (Higginbotham and Ramchand, 1997; Fernald, 2000). Moreover, it is also not clear why (14-b) should be false and unacceptable, as the property is 'temporal' here (and the truth conditions in (4) are satisfied).

However, pursuing a line of investigation that takes into account temporal considerations seems to be the right direction, as revealed by the following observations.

\subsection{New observations on asymmetric relations}

In the following discussion, we rely on the distinction between permanent and nonpermanent relations denoting predicates. We adopt a pragmatic view of this distinction, in the spirit of Condoravdi (1992:9). ${ }^{8}$ Let $T$ be the set of times, $\prec$ a precedence relation, and $t_{1} \prec t_{2} \prec t_{3} \prec \ldots \in T$. A predicate that denotes a permanent relation $R$ is associated with an inference persistence that specifies what follows. For any $x, y \in A$ (with $x \neq y$ ), if $x$ stands in the relation $R$ with $y$ at time $t_{1}$ and you have no information that $x$ does not stand in the relation $R$ with $y$ at time $t_{2}$, then infer that $x$ stands in the relation $R$ with $y$ at time $t_{2}$. As Condoravdi (ibid.) notes, this is a default inference that surfaces only when there is no information to the contrary.

In the remainder of this paper we no longer mention the 'inclusive alternative orderings' interpretation, as the truth conditions provided by Dalrymple et al. (1998) in (4) are immune from temporal considerations.

In this section, we reconsider constraints on asymmetric relations, revisiting the facts discussed in Beck (2001) in the light of the permanent / non-permanent distinction.

Firstly, comparatives are out only when they denote a permanent relation in the context of utterance (see (18-a) vs (18-b)).

a. We act smarter than each other. ${ }^{9}$

\footnotetext{
${ }^{8}$ See also McNally, 1993 and Magri, 2009. Condoravdi talks about eventualities occurring in time. As we do not adopt an ontology including eventualities, we modify the definition provided by the author accordingly.

${ }^{9}$ http://www.respectrx.com/archives/selfrespect_selfesteem/all_this_and_brains_too. html
} 
b. \#We are smarter than each other.

There are two types of situations in which (18-a) is judged true. In the first one, each participant acts once, and each participant acts smarter than the previous participant ${ }^{10}$. In the second one, each participant acts many times, and each time that a participant acts, s/he acts smarter than the preceding participant.

The same observation holds for abilitative readings. The English present tense is well known to yield a generic interpretation (e.g., Kenny, 1963; Krifka et al. 1995; Chierchia, 1995; Menendez-Benito, 2005). In this interpretation, (19-a) ascribes to each man the ability to run at a speed that differs from the speed at which every other man in the set can run. Abilities are permanent properties of men, and the sentence is unacceptable.
a. \#These men run faster than each other (generic ability interpretation).
b. These men run faster than each other at each lap.
c. These men are running faster than each other at each lap.

When a competition scenario is provided, the sentence is rescued, as in (19-b). The sentence improves even more if the progressive form is used (19-c). ${ }^{11}$

As signaled by the adverbial at each lap, (19-b) is about actual runnings and not generic abilities. Sentence (19-c) is also about actual runnings, as the progressive is never used on a generic reading (Dahl, 1975; Krifka et al. 1995). There are various types of scenarios where these sentences are judged true: (i) every man runs one lap only and faster than the preceding man; (ii) every man runs more than one lap and faster than the preceding man; (iii) all men run at the same time, they run many laps and a different man wins at each lap.

Secondly, we note that, in some cases, reciprocal sentences with geometric predicates are unacceptable, as in (20). In this case, the permanency of the relationship between the head and body seems again responsible for the unacceptability of the sentence. In fact, (20) is compatible with each other in a context wherein the head has been decapitated from the body.

(20) Scenario: a living human being is being described.

\# The head and the body are on top of each other.

\footnotetext{
${ }^{10}$ The cases in which each member of the reference set is involved once in the reciprocal relation are discussed in section 5.4 .

${ }^{11}$ While (i) is mostly avoided if it is taken to express that each American president is younger than the preceding one, it is more easily accepted under the interpretation that each American president is younger than the preceding one at the time when he becomes president. Remarkably, though, those who accept the sentence clearly prefer it with the addition of 'all.' As 'all' is a floating quantifier, a separate study of its interaction with each other would be required. Such a study is outside the scope of this paper (for insights, see Schwarzschild, 1996).
}

(i) (??)The successive American presidents were all younger than each other 
The immediate question that these data raise is why permanency should play a role in the interpretation of reciprocal each other-sentences with asymmetric relations.

We must note, however, that appealing to non-permanency does not suffice to tease apart the sentences in (21).

a. Scenario: the older king dies and then the younger one succeeds. \# Two kings succeeded each other.

b. Two boys followed each other.

In both (21-b) and (21-a), the relation between the two members is non-permanent. Still, only (21-b) is acceptable.

Barry Schein (p.c.) has suggested to us another example in which the relation is nonpermanent and the sentence is unacceptable. Assume a restaurant in which pizzas are delivered in stacks, with pepperoni pizzas always stacked on mushroom pizzas. In this scenario, (22) is hardly used. Conversely, if the pizzas are stacked without a precise rule, (22) can be used, even if it turns out that the pepperoni pizzas are all stacked on the mushroom pizzas.

$(\#)$ The mushroom pizzas and the pepperoni pizzas are stacked on top of each other.

\subsection{Descriptive conclusions}

The data show that comparatives and geometrical asymmetric predicates are allowed in reciprocal each other-sentences only if they denote a relation that does not permanently hold. However, that the relation holds non-permanently is not always sufficient to guarantee that reciprocal each other-sentences are acceptable with asymmetric relations.

The endeavor of a proper reciprocal theory is to explain why non-permanency is necessary with asymmetric predicates.

Moreover, such a theory must explain the common link between the unacceptability of the cases in which the relation is permanent (i.e., (20), (18-b), (19-a)) and those in which in which it is non-permanent (i.e., (12), (21-a), (22)). ${ }^{12}$

\footnotetext{
${ }^{12} \mathrm{~A}$ final piece of data that pertains to asymmetric relation but is peripheral to the main claim of this paper pertains to the well-known question of directionality (Langendoen, 1978).

(IIa) These men succeeded/\#preceded each other.

(IIb) The tables are stacked on top of/\#beneath each other.

Initially, Langendoen (ibid.) explains these differences using general cognitive principles: sequences develop from left to right and bottom-up. Schein (2003) has noted, however, that contextual information can overwrite these principles and that, for instance, (III) is true in a scenario where a pile of plates hangs down from a shelf, with each plate stacked to the one on top by a magnet.

(III) Scenario: a pile of plates is hanging down from a shelf.

The plates are stacked beneath each other. (Schein, 2003)

This paper does not have much to add to the debate. Leaving the debate where it stands, we only note that across a variety of categories, antonyms, such as on top of / beneath, in natural language do not have
} 
For convenience, we repeat here the sentences that a proper theory of reciprocals must rule out, distinguishing between the cases in which the relation is permanent (23) and those in which the relation is non-permanent (24).

(23) The predicate denotes a permanent relation.

a. Scenario: a living being is being described.

\# The head and the body are on top of each other $(=(20))$.

b. \#They are smarter than each other $(=(18-\mathrm{a}))$.

c. \#These men run faster than each other $(=(19-\mathrm{a}))$ (Abilitative interpretation).

(24) The predicate denotes a non-permanent relation.

a. \#My mother and I gave birth to each other $(=(12))$.

b. Scenario: a king cannot succeed his successor.

\# The two kings succeeded each other $(=(21-\mathrm{a}))$.

c. Scenario: in a restaurant the habit is to always stack the mushroom pizzas on the pepperoni pizzas.

\#The mushroom pizzas and the pepperoni pizzas are stacked on top of each other $(=(22))$.

While the contrasts involving permanent and non-permanent properties have not been previously considered in detail, the thesis that reciprocal sentences are parametric to time has already been investigated. ${ }^{13}$

Theories that have resorted to time can rule out sentences in (23), i.e., those sentences in which the predicate denotes a permanent relation. However, accounts uniquely based on time cannot capture cases in (24), in which the predicate denotes a relation that holds non-permanently but the sentence is nonetheless unacceptable.

We comment on theories using time and explain why this is not sufficient to capture the observations in section 6, where we discuss the theories of Schein (2003) and Beck and von Stechow (2007).

In the next section we present our own theory that resorts to worlds and times.

the same status as one another. Consider, for instance, the tall/short pair. Tall is the neutral antonym, as the following contrast shows: 'Susan is as tall as Walt' $\nrightarrow$ 'Susan and Walt are tall' vs. 'Susan is as short as Walt' $\rightarrow$ 'Susan and Walt are short.' Across languages, it seems that the antonym chosen as neutral is the positive one. For the pair on top of / beneath, the positive one on top of would be chosen by default, while beneath would be used in specific contexts such as that pointed by Schein (ibid.) The investigation of the characteristics of these neutral terms is beyond the scope of this paper (Winter, 2009 presents a recent discussion).

${ }^{13}$ The first who consider time within a theory of reciprocals are Fiengo and Lasnik (1973). 


\section{Reciprocity, worlds and times}

\subsection{Strong reciprocity and reasonable worlds}

To account for the restrictions on the use of each other, we claim that reciprocal each othersentences are true and acceptable if the relation is either actually or possibly strongly reciprocal on the reference set, provided that the possibilities (including the actual world) are 'reasonable'.

Before considering the notion of 'reasonability', let us say a little more about possibilities and the actual world. In our account, we consider time-world coordinates and use sets of times and worlds. We assume that, for any given time, the past of a world at that time is unique, whereas the not-yet-realized-future of that world at that time, is one of the options available at $t .^{14}$

In particular, considering the utterance time $t_{0}$, we assume that the actual world up to and including $t_{0}$ is unique but that there is not yet an actual world after $t_{0}$ and that a variety of options are available.

For any time $t$, we call a 'reasonable world-to-come' at $t$, a 'reasonable future' fixed at $t$. In our framework, the time $t$ at which reasonable futures are considered can either coincide with or precede the utterance time. It is possible that the actual world-to-come at $t$ belongs to the set of reasonable futures fixed at time $t$.

The hypothesis we make to account for the restrictions on the use of each other thus is rephrased as follows: 'A reciprocal each other-sentence is true if the relation is strongly reciprocal across reasonable futures fixed at a time $t$ '.

Let us introduce reasonable futures via the following example (25).

$$
\text { \#The Eiffel Tower and the Empire State Building are taller than each other. }{ }^{15}
$$

We state that this sentence is false and infelicitous because strong reciprocity cannot be enacted. More precisely, strong reciprocity cannot be enacted in those futures (fixed at $t_{0}$ or at a time preceding $t_{0}$ ), in which the sizes of the Eiffel Tower and of the Empire State Building do not change (and are such that the Eiffel Tower becomes taller than the Empire State building, thus obtaining strong reciprocity). These are the futures that are considered for the evaluation of the reciprocal sentence (25).

The futures that one considers when evaluating a reciprocal sentence are called 'reasonable'. What is a reasonable future? For the particular case (25), what are the futures in which sizes do not change?

A reasonable future determined at time $t$ is such that the rules that hold at $t$ are maintained. Rules can be social (determined by convention), natural (the laws of nature),

\footnotetext{
${ }^{14}$ We present the formal framework of branching time in section 3.2.

${ }^{15}$ For the sake of the example, we need to know that the Eiffel Tower was completed in 1889 and measures 324 meters. The Empire State Building was completed in 1931 and measures 381 meters.
} 
or moral (laws that are conform to the commonly admitted accepted moral behavior). They also include habits (i.e. behavior patterns). Rules, especially social ones, can be restricted to very specific contexts as well (e.g., rules that hold in my office, my home, and my building).

Consider (25). The rule that matters when interpreting the sentence can be summarized as, 'Once completed, skyscrapers do not change size'. When evaluating this sentence, one disregards those futures in which attacks, earthquakes and similar aberrant events occur (see Dowty's 1979 notion of inertia and discussion infra), which would cause the Empire State Building to become shorter than the Eiffel Tower. Note that, because social, moral and even natural aberrations occur very often (see discussion in Landman, 1992; Kagan, 2011), the world that will become the actual world could never belong to the set of reasonable futures of any time. When evaluating sentence (25), those aberrations that would affect the individuals in the reference set are considered (e.g., when interpreting (25), one does not consider an earthquake occurring in Asia, as this, presumably, would not modify the size of the Empire State Building).

Moreover, when interpreting (25), one also disregards futures in which aberrations (such as attacks and earthquakes) do not occur but in which the rules that hold at the time at which reasonable futures are considered are nonetheless modified. For example, when evaluating (25) at time $t_{0}$, one disregards those futures in which the French government decides to add floors to the Eiffel Tower so that it becomes the highest building in the world. Adding floors would modify the rule that is in place at the utterance time, which is 'Once completed, skyscrapers do not change their sizes'. ${ }^{16}$

Reasonable worlds are such that also habits and behaviors do not change there. Of course habits can change. However, those worlds in which they change are discarded.

Note that selecting futures, in which the rules that hold in the actual world at the time at which futures are considered also hold, is a specific requirement associated with each other sentences in English. Future investigation of reciprocals in other languages will show whether a similar notion of reasonableness is also needed and whether and to what extent it can be at play for other expressions as well. Let us note, however, that this notion may be useful at least for the analysis of the progressive in English. The following notes compare the notion of reasonability and Dowty's notion of inertia.

When introducing the notion of inertia worlds, Dowty (Dowty, 1979, p. 148) advocates the 'natural course of events' and explains (ibid.) 'these are to be thought of as worlds which are exactly like the given world up to the time in question and in which the future

\footnotetext{
${ }^{16}$ The futures in which the French government decides to add floors to the Eiffel Towers can be taken into account when 'plans' are considered. In this case, the future will is used. See discussion in section 5.2. Note also that, in a context in which the Empire State Building is under construction, in 1930, the Eiffel Tower and the Empire State Building were taller than each other at different stages of the construction of the Empire State Building is true and acceptable. In this case the modifier 'at different stages of the construction of the Empire State Building' is mandatory (see section 5.2).
} 
course of events after this time develops in ways most compatible with the past course of events.'

The ways most compatible with the past course of events exclude interferences. Dowty considers the following example (number (26) in Dowty, 1979, p. 149):

John is ruining his academic reputation by publishing all those crackpot papers on politeness in Pre-Indo-European.

Dowty (ibid., p. 49) explains that regardless of whether someone will interfere and suggest that John publish profound ideas on a different topic, what is at stake is what is happening now, "what is the outcome of events as they could be expected to transpire without such interferences' (Dowty, ibid., p. 149).

Landman's exegesis of Dowty (Landman, 1992) also presents the notion of inertia in terms of normality conditions (Landman (ibid.), p. 3): "these worlds are to be understood in the following way: the inertia worlds for our world at an interval are identical to our world up to that interval. From there on they are the worlds in which nothing unexpected happens, in which everything takes its natural, normal course.' This view nicely applies to a case like (27) (example (12) in Landmann (ibid.), p. 10). According to Dowty's notion of normality as inertia, the only worlds considered are those in which the thunderbolt does not strike Mary.

Mary was crossing the street, when a thunderbolt from heaven struck her down

Our notion of reasonability is different from Dowty's notion of inertia and does not appeal to what is 'normal' given the present course of events. Consider again example (25) in a context in which a meteorite falls on the Empire State Building on December 2012 and destroys half of it, rendering it shorter than the Eiffel Tower. The meteorite has been flying around the earth for a while. (25) is unacceptable even in this scenario. Nonetheless, if we were to employ the notion of inertia, it would wrongly be predicted acceptable ${ }^{17}$, as the falling of the meteorite is part of the world at 'now' and is part of the normal course of events.

Reasonable futures are those in which habits/laws/behaviors are not interrupted by any interference and not of worlds that are the most 'compatible' with the actual world at a given time, as in those worlds, habits/laws/behaviors can be interrupted after 'now' by factors that are already part of the actual world at 'now'. Our notion of reasonability is reduced to the total absence of interferences that would disrupt the course of the law/habit/behavior that holds at the time at which reasonable futures are considered. Only those worlds in which, no matter what, laws/habits/behaviors that hold at the time

\footnotetext{
${ }^{17}$ In this scenario, the Empire State Building is taller than the Eiffel Tower during the period of time comprised between its completion and the falling of the meteorite. It is shorter than the Eiffel Tower after the falling of the meteorite. Strong reciprocity is thus obtained.
} 
at which reasonable futures are considered continue to hold, are reasonable.

Related to the semantics of the progressive in English, Landman (1992, crediting Vlach, 1981) had already raised a criticism similar to ours of the notion of inertia. Landman explains that Dowty's view runs into problems when it comes to a case such as the following (28) (example (13) in Landman (ibid.), p. 10).

Mary was crossing the street, when the truck hit her.

On Dowty's view, the event of Mary crossing the street continues in worlds that are most compatible with the actual world of 'now', i.e., in those worlds in which nothing unexpected happens. As Landman points out, such interferences or unexpected things are, sometimes, already part of the world at 'now' and are thus part of the normal course of events. In the scenario that the author proposes for (28), the truck is approaching Mary while she is crossing the street (Landman ibid., p. 10). The arrival of the truck is part of the normal course of events that includes Mary crossing the street, and only a miracle could save Mary even in an inertia world. Landman (ibid.) uses this example to justify a theory for the progressive that uses events. ${ }^{18}$

Because we do not use events in our theory of reciprocals, we do not consider Landman's proposal here. We nonetheless suggest that consideration of reasonable futures, i.e. of those futures in which no factors orthogonal to the law/habit/behavior considered intervene and laws/habits/behavior steadily hold, might turn out to be useful for the analysis of the progressive in English as well.

Let us further emphasize that the actual world to come after the utterance time can belong to the set of reasonable worlds fixed at $t_{0}$. Because the actual world after the utterance time does not yet exist and because nothing guarantees that it will be reasonable, the constraint that only reasonable futures should be considered hinges on available continuations of the actual world for a fixed time $t$ that either precedes or coincides with the utterance time.

The following section elaborates the modal framework that we employ.

\subsection{The framework}

\subsubsection{Basic notions}

We employ a $W \times T$ forward-branching structure (Thomason, 1984). A three-place relation $\simeq$ on $T \times W \times W$ is defined such that (i) for all $t \in T, \simeq_{t}$ is an equivalence relation;

\footnotetext{
${ }^{18}$ Landman proposes a theory in which what is internal to an event is considered and poses two constraints on progressive sentences in English: (i) consideration of events that have reasonable chances of continuing if interrupted in the actual world and (ii) consideration of those non-actual worlds in which a given event (e.g., Mary crossing the street) continues (in virtue of what this event is, i.e., in this case, an event of crossing the street such that the person crossing the street reaches the other side) if interrupted in the actual world.
} 
(ii) for any $w, w^{\prime} \in W$ and $t, t^{\prime} \in T$, if $w^{\prime} \simeq_{t^{\prime}} w$ and $t$ precedes $t^{\prime}$, then $w^{\prime} \simeq_{t} w$ (we use the symbols $\prec$ and $\succ$ for temporal precedence and succession, respectively). In words, $w$ and $w^{\prime}$ are historical alternatives at least up to $t^{\prime}$ and thus differ only, if at all, in what is future to $t^{\prime}$.

Assume two worlds $w^{\prime}$ and $w$ in $W$ and two times $t^{\prime}, t^{\prime \prime}$ in $T$ such that $t^{\prime} \prec t^{\prime \prime}$. In both partial models in Figure 1, $w^{\prime}$ and $w$ are equal up to and including $t^{\prime}$ (Thomason, 1984). Worlds that stand in the equivalence relation $\simeq_{t^{\prime}}$ need not branch at $t^{\prime}$; they can branch at a time after $t^{\prime}$ (e.g., $t^{\prime \prime}$ in Figure $\left.1 \mathrm{~b}\right)$.

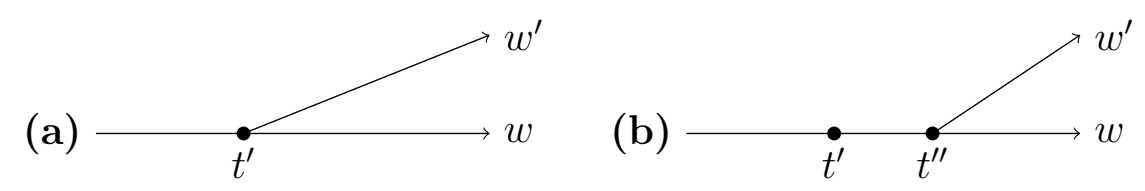

Figure 1: $w \simeq_{t^{\prime}} w^{\prime}$

As Condoravdi (2002) explains, for any given time, a world belongs to an equivalence class comprising worlds with identical pasts but different futures. Let $w_{0}$ be the actual world.

Figure 2 depicts the two equivalence classes in (29), induced by the relations $\simeq_{t 1}$ and $\simeq_{t 2}$.
a. $\quad w_{0} \simeq_{t_{1}} w_{1} \simeq_{t_{1}} w_{2} \simeq_{t_{1}} w_{3} \simeq_{t_{1}} w_{4}$
b. $\quad w_{0} \simeq_{t_{2}} w_{2} \simeq_{t_{2}} w_{3}$

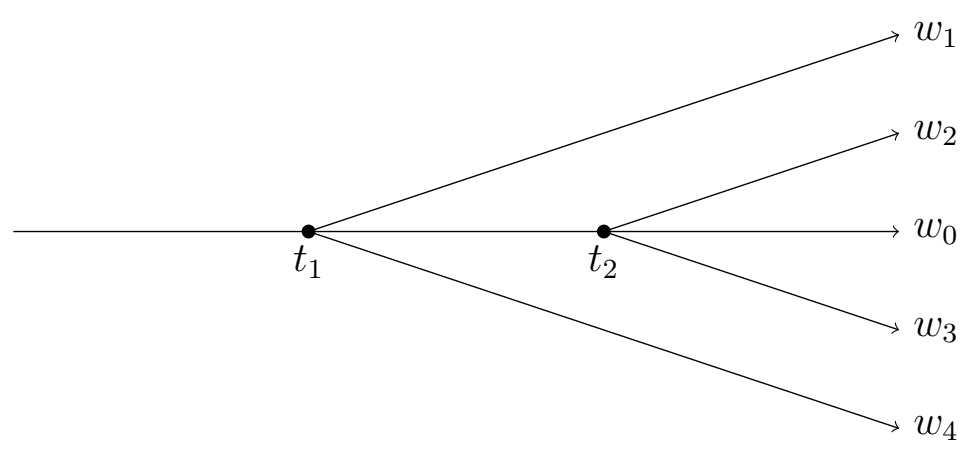

Figure 2: Equivalence classes of worlds

\subsubsection{Common ground and reasonable futures}

For any time $t \in T$, we define the common ground $c g(t)$ as the set of worlds that are identical to the actual world $w_{0}$ at least up to and including $t$.

$$
\operatorname{cg}(t):=\left\{w \mid w \simeq_{t} w_{0}\right\}
$$


In the case depicted in Figure 3, the common ground at $t$ is the set given in (31).

$$
c g(t)=\left\{w_{1}, w_{2}, w_{0}, w_{3}, w_{4}\right\}
$$

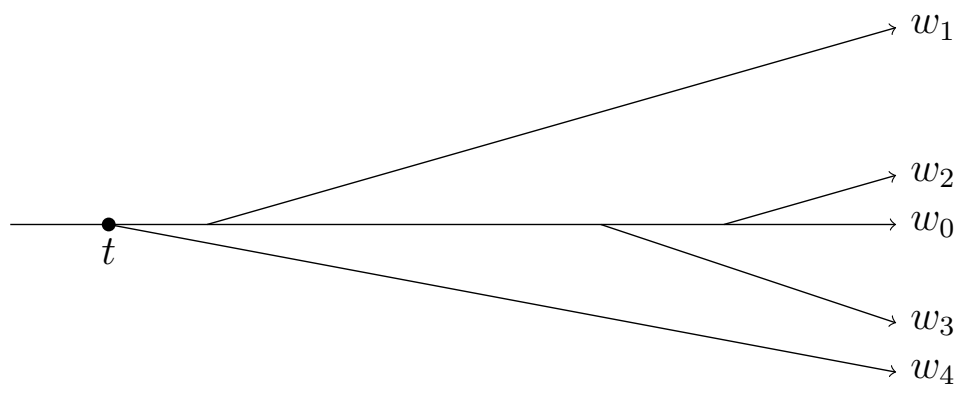

Figure 3: $c g(t)$

So defined, the common ground includes any world branching from the actual world at a time equal to or after $t$, including those worlds that are highly different in their causal laws from the actual world as well as those worlds in which current causal and social laws malfunction.

In this framework, 'reasonable futures', are defined as in (32). For any $t \in T$,

$$
\begin{aligned}
& \text { ReasFut }(t):=\left\{w_{i} \in c g(t) \mid w_{i} \text { is such that the set of rules fixed at } t\right. \\
& \text { continue to hold in } \left.w_{i}\right\}
\end{aligned}
$$

Let us assume that $w_{3}$ in Figure 3 is a world in which causal and social laws malfunction. The set of reasonable futures defined at time $t$ does not include $w_{3}$.

In the case depicted in Figure 4, the set of reasonable futures fixed at $t$ is given in $(33)$.

$$
\operatorname{ReasFut}(t)=\left\{w_{1}, w_{2}, w_{0}, w_{4}\right\}
$$

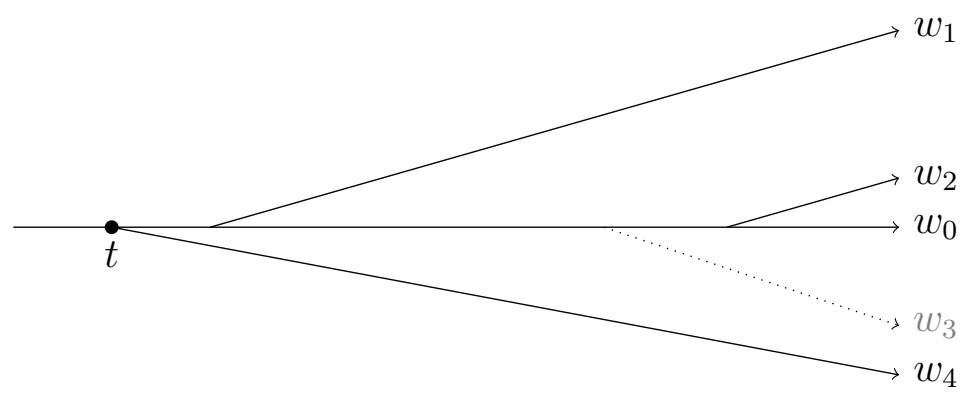

Figure 4: Reasonable Future Worlds (ReasFut) 
Let us add an important remark. As it stands, picture 4 could only be drawn by an omniscient god, as the actual world $w_{0}$ is not an already existing complete history. Let us assume more specifically that $t$ is a time that either precedes or coincides with the utterance time $t_{0}$. At time $t$, one can state what the reasonable futures of time $t$ are, however, one cannot state whether the actual-world-to-come belongs to the set of reasonable possibilities, since the latter does not exist yet at $t$.

Let us illustrate this via the example in (25). Let $t \prec t^{\prime} \prec t^{\prime \prime}$ be three times in the set $T$. A set of reasonable worlds is determined at $t$, which for (25) is the time at which the construction of the Empire State Building is completed. Consider the world $w_{0}$ at $t^{\prime}$. At this time, no earthquakes have occurred, and neither the American nor French government has added or suppressed floors (see Figure 5).

The actual world $w_{0}$ coincides with world $w_{1}$ until at least $t^{\prime}$ (the ' $=$ ' sign in Figure 5 and 6 is to be read 'coincides with'). The branch that represents the actual world is in bold in the two figures that follow.

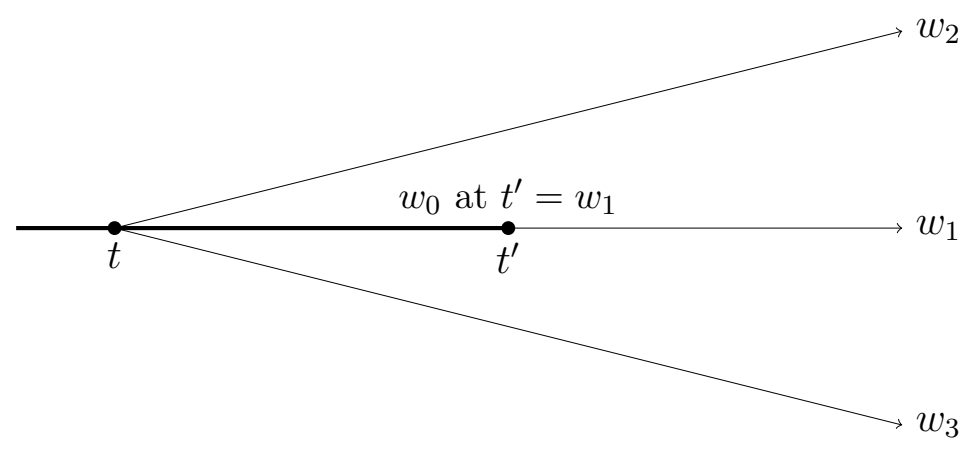

Figure 5: The actual world at $t^{\prime}$ coincides with a reasonable future determined at $t$

Between $t^{\prime}$ and $t^{\prime \prime}$, an earthquake occurs in $w_{0}$ in New York, destroying part of the Empire State Building. From that moment on, the actual world follows a branch $w_{4}$, which is not part of the reasonable futures of $t$ (see Figure 6 , where $w_{4}$ is marked by a dotted line).

In this scenario, the set of rules fixed at $t$ continues to hold in $w_{0}$ until at least $t^{\prime}$ and the actual world $w_{0}$ coincides until $t^{\prime}$ with an element of the set ResFut $(t)$.

With this example in mind, let us consider the actual world at the utterance time. At the utterance time, to say that $w_{0} \in \operatorname{ResFut}(t)$ (with $t \preceq t_{0}$ ) is improper because one would have to know, at the utterance time, what the not-yet-realized actual world is like. But the actual world exists only until the utterance time.

Still, by abuse of notation, for simplicity, we write $w_{0} \in \operatorname{ResFut}(t)$ meaning that the actual world has coincided with an element of ResFut $(t)$ until $t_{0}$ or, more simply, that the laws that hold at $t$ continue to hold in the actual world until at least the utterance time. 


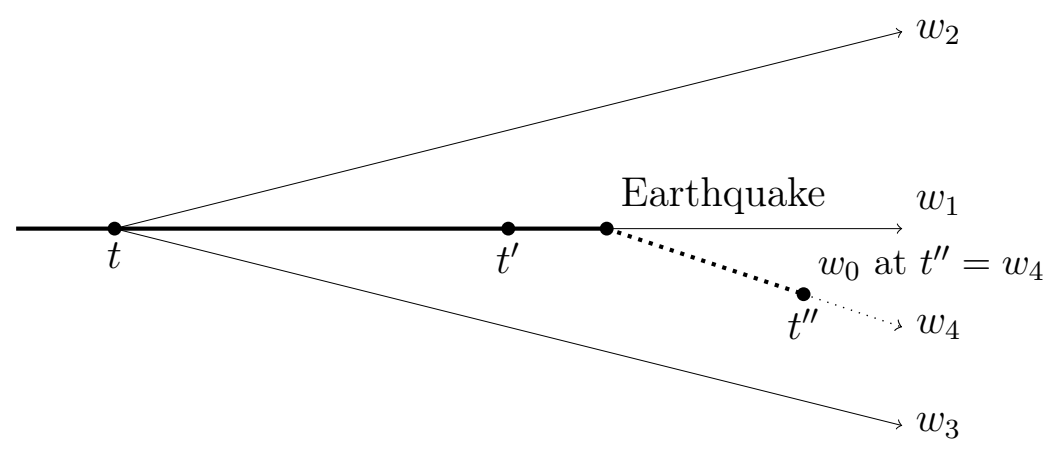

Figure 6: The actual world at $t^{\prime \prime}$ does not coincide with a reasonable future determined at time $t$

Let us note to conclude that the way we use branching time here is equivalent to a system based on world-time indices, together with a specification of which world is equal to which other world up to what time, plus consideration of accessibility relations (Kratzer, 1991). In a framework taking into account world-time indices, we would then specify that only worlds that satisfy the same laws and habits in vigor in the actual world are accessible from the actual world at the time at which they cease to be identical to the actual world. It is thus possible to translate the theory that we are going to propose in terms of worldtime indices, plus specification of appropriate accessibility relations. The purpose of using branching time here is purely practical, as it provides very convenient tools for expressing the notions we need for our story about reciprocals (see related discussion in Dowty (1979:153)).

With this temporal and modal set up, we now turn to the interpretation of each other sentences.

\subsection{Truth conditions for each other sentences}

Following Keenan (1987), Dalrymple et al. (1998), Winter (2001), Dotlačil and Nielsen (2008), and Sabato and Winter (2011), as we mentioned it in the introduction, we consider each other to be a polyadic quantifier. The tense phrase denotes the reciprocal relation $R .^{19}$

Let us fix $R$ and $A$. $A$ stands for a plural (i.e., non-singleton) set and $R$ for a binary relation over $A \times A$. We write as $A \backslash\{x\}$ the set $A$ without the element $x$.

Any element $t$ of the set $T$ is a precise, discrete moment. So intervals, which are subsets of $T$ are sets of moments. In particular, for any $t \in T,\{t\}$ is the interval (formally, the subset of $T$ ) that is reduced to the singleton $t$. For all $t \in \mathcal{T}$, we write $R(\langle x, y\rangle, w,\{t\})$ whenever $x$ stands in relation $R$ with $y$ at time $t$ in world $w$. On another hand, $(\neg R)(\langle x, y\rangle, w,\{t\})$ means that $x$ doest not stand in relation $R$ with $y$ at time $t$ in

\footnotetext{
${ }^{19}$ By 'tense phrase' we mean the extended verbal projection together with tense and aspect.
} 
world $w$.

For any interval $\mathcal{Z}$ and any world $w \in W, R(\langle x, y\rangle, w, \mathcal{Z})$ means that $x$ stands in relation $R$ with $y$ at the interval $\mathcal{Z}$ in world $w ;(\neg R)(\langle x, y\rangle, w, \mathcal{Z})$ means that $x$ does not stand in relation $R$ with $y$ at interval $\mathcal{Z}$ in world $w$. Let us define these notions more accurately, according to the different types of predicates.

For any atelic verb phrase ${ }^{20}, R(\langle x, y\rangle, w, \mathcal{Z})$ means that for any time $t \in \mathcal{Z}, R(\langle x, y\rangle, w,\{t\})$, which implies that $\forall \mathcal{N} \subset \mathcal{Z}, R(\langle x, y\rangle, w, \mathcal{N})$. All the same, $(\neg R)(\langle x, y\rangle, w, \mathcal{Z})$ means that for any time $t \in \mathcal{Z},(\neg R)(\langle x, y\rangle, w,\{t\})$, and implies in turn that $\forall \mathcal{N} \subset \mathcal{Z},(\neg R)(\langle x, y\rangle, w, \mathcal{N})$. Note that $(\neg R)(\langle x, y\rangle, w, \mathcal{Z})$ is not equivalent to $\neg(R(\langle x, y\rangle, w, \mathcal{Z}))$, which only means that $\exists t \in \mathcal{Z},(\neg R)(\langle x, y\rangle, w,\{t\})$. We call $(\neg R)(\langle x, y\rangle, w, \mathcal{Z})$ the negative condition of $R(\langle x, y\rangle, w, \mathcal{Z})$.

For any achievement verb phrase, the relation is verified only at a precise moment, so we set $\mathcal{Z}$ to $\{t\}$, where $t \in \mathcal{T}$. In this case, $R(\langle x, y\rangle, w, \mathcal{Z})$ rewrites as $R(\langle x, y\rangle, w,\{t\})$ and $(\neg R)(\langle x, y\rangle, w, \mathcal{Z})$ reads $(\neg R)(\langle x, y\rangle, w,\{t\})$.

Finally, the definition of $R(\langle x, y\rangle, w, \mathcal{Z})$ and $(\neg R)(\langle x, y\rangle, w, \mathcal{Z})$ might appear as problematic for accomplishments, on the assumption that these do not have the subinterval property (i.e. whenever $R$ holds at interval $\mathcal{Z}$, it does not hold at any subinterval of $\mathcal{Z}$ (see Bennett and Partee, 1972)). On this view, $R(\langle x, y\rangle, w, \mathcal{Z})$ implies that $\exists \mathcal{N} \subset$ $\mathcal{Z}(\neg R)(\langle x, y\rangle, w, \mathcal{N}) . \quad(\neg R)(\langle x, y\rangle, w, \mathcal{Z})$ also implies that $\exists \mathcal{N}^{\prime} \subset \mathcal{Z}(\neg R)\left(\langle x, y\rangle, w, \mathcal{N}^{\prime}\right)$. Nonetheless, even on this view, $R(\langle x, y\rangle, w, \mathcal{Z})$ and $(\neg R)(\langle x, y\rangle, w, \mathcal{Z})$ remain incompatible if we argue that (i) the negation of a telic predicate is an atelic one (e.g. not eat an apple is atelic), so $(\neg R)(\langle x, y\rangle, w, \mathcal{Z})$ implies that $\forall \mathcal{F} \subset \mathcal{Z}(\neg R)(\langle x, y\rangle, w, \mathcal{F})$, and (ii) $R(\langle x, y\rangle, w, \mathcal{Z})$ implies that $\exists \mathcal{F} \subset \mathcal{Z}(R)(\langle x, y\rangle, w, \mathcal{F})$. Presumably, this is at least the case for $\mathcal{F}=\{z\}$, where $\{z\}$ is the right bound of $\mathcal{Z}$.

How to define accomplishments otherwise than appealing to the lack of the subinterval property is a very complex matter that we do not address here (see, e.g. Dowty, 1979; Bach, 1986; Krifka, 1988; Rothstein, 2004,2008).

Let $t_{0}$ be the utterance time. (34) provides the truth conditions for each other sentences. A paraphrase follows the formal rule.

$$
\begin{aligned}
& E O(A, R) \text { is true at } w_{0}, t_{0} \text {, iff } \\
& \text { (i) } \exists \mathcal{I}=[a, b] \text { s.t. } t_{0} \in \mathcal{I} \& w_{0} \in \operatorname{ReasFut}(a) \text { \& } \\
& \text { (ii) } \exists x \in A \exists y \in A \backslash\{x\}\left(R\left(\langle x, y\rangle, w_{0}, a\right)\right) \& \\
& \text { (iii) } \forall x \in A \exists y \in A \backslash\{x\} \exists \mathcal{J} \subseteq \mathcal{I}\left(R\left(\langle x, y\rangle, w_{0}, \mathcal{J}\right) \vee R\left(\langle y, x\rangle, w_{0}, \mathcal{J}\right)\right) \& \\
& \text { (iv) } \forall x \in A \forall y \in A \backslash\{x\} \exists c \succeq b \exists \mathcal{K} \subseteq[a, c] \exists w_{i} \in \operatorname{ReasFut}(a),\left(R\left(\langle x, y\rangle, w_{i}, \mathcal{K}\right)\right) .
\end{aligned}
$$

Let us provide a line-by-line paraphrase with some comments.

An each other-sentence is true in the actual world at utterance time $t_{0}$ if and only if:

\footnotetext{
${ }^{20}$ Given our syntax-semantic interface here, we assume that Vendler's classification applies at the level of the VP.
} 
(i) there is an interval $\mathcal{I}=[a, b]$ such that $t_{0} \in \mathcal{I}$, and the set of rules fixed at a continue to hold in the actual world until at least $t_{0}$.

Since we are adopting a temporal-modal framework, we must take care of the time at which the reciprocal relation holds. As it stands, (i) condition holds for non-past reciprocal each-other sentences. For past reciprocal sentences, condition (i) is as follows:

(i') $\exists \mathcal{I}=[a, b]$ s.t. $b \prec t_{0} \&$ at $b$, the world $w_{0} \in \operatorname{ReasFut}(a)$

In this case the utterance time follows the time $b$ and, when evaluated at $b$, the actual world belongs to the set of reasonable futures determined at time $a$.

(ii) In the actual world, there is an ordered pair of elements $\langle x, y\rangle$ (with $x \neq y$ ) that stand in the relation $R$ at the origin $a$ of the interval $\mathcal{I}$.

The origin of $\mathcal{I}$, i.e., time $a$, is generally considered to be the first time at which two elements in the reference set stand in the relation $R$.

(iii) In the actual world, every element $x \in A$ is or has been involved in the relation $R$ with some element $y \in A$ (either as the first or second element of the ordered pair), in $\mathcal{I}$ or in a subinterval $\mathcal{J}$ of $\mathcal{I}$. For every pair of individuals, the choice of the interval at which they stand in the relation $R$ is made independently from other pairs.

Note that this condition holds for $w_{0}$, up to time $b$ (with $b \preceq t_{0}$ ). In this case, one is allowed to consider the actual world because (i) guarantees that $w_{0}$, up to $t_{0}$, belongs to the set of reasonable futures of $a$.

(iv) Every ordered pair $\langle x, y\rangle$ (with $x \neq y$ ) is involved in the relation $R$ in a reasonable future of time $a$, in a subinterval $\mathcal{K}$ of an interval $[a, c]$ that can be larger than $\mathcal{I}$. This condition acknowledges that strong reciprocity can be enacted after $t_{0}$ (i.e., after time $b \in \mathcal{I}$, with $\left.b=t_{0}\right)$. Again, because the actual-world-to-come does not yet exist at $t_{0}$ and because one cannot know whether it will be reasonable, the constraint that strong reciprocity be enacted holds for reasonable futures determined at $a$.

For every pair of individuals $\langle x, y\rangle$ (with $x \neq y$ ), the choice of the reasonable future at which they stand in the relation $R$ is made independently of how it is made for other pairs of individuals, given that the universal quantifiers binding individuals out-scope the existential quantifiers over reasonable futures and intervals.

In a nutshell, the truth conditions require that, by the utterance time, all the elements are or have been involved in the relation $R$ either as the first or second argument and that strong reciprocity be enacted across reasonable futures determined at the origin of the interval $\mathcal{I}$, which, if it is reasonable, also include the actual world-to-come after $t_{0}$.

The following section describes the possibilities allowed by the rule.

\subsection{Examples}

In the examples that follow we call $\mathcal{F}$ the interval that starts at time $a$ and ends at time $c$ (with $c \succeq b$ ). 
Granted that conditions (i)-(iii) are satisfied, condition (iv) requires that strong reciprocity be enacted across reasonable futures of time $a$. It can thus be enacted at $\mathcal{F}$ in the actual world, if this belongs to the set of reasonable futures of $a$ after $t_{0}$.

As noted above, at $t_{0}$, one cannot know whether the not-yet-occurred-actual-world coincides with one of the reasonable futures determined at time $a$. In the cases discussed below, we assume that it does.

Note again that, for each pair, the choice of the interval and of the reasonable future is made independently of the other pairs.

1. In Figure 7, strong reciprocity is enacted at $\mathcal{F}=[a, c]$. In this case, all pairs $\langle x, y\rangle$ in $A \times A$ (with $x \neq y$ ) stand in the relation $R$ at $\mathcal{K}_{1}=\mathcal{K}_{2}=\mathcal{F}$ in the actual world. ${ }^{21}$ For each pair, the rule requires that there is a dedicated interval at which they stand in relation $R$. As there are two pairs, we distinguish between $\mathcal{K}_{1}$ and $\mathcal{K}_{2}$. Both are equal to $\mathcal{F}$.

(35) The two men know each other.

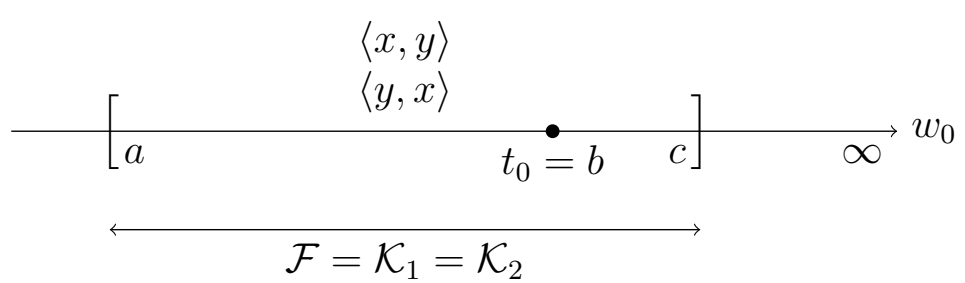

Figure 7: Strong reciprocity in $w_{0}, \mathcal{F}$, without consideration of subintervals

2. In Figure 8, the two pairs $\langle x, y\rangle,\langle y, x\rangle$ (with $x \neq y$ ) stand in the relation $R$ at two different subintervals $\mathcal{K}_{1}, \mathcal{K}_{2} \subseteq \mathcal{F}$, with $\mathcal{K}_{1}$ and $\mathcal{K}_{2}$ overlapping each other.

(36) The two men are hitting each other.

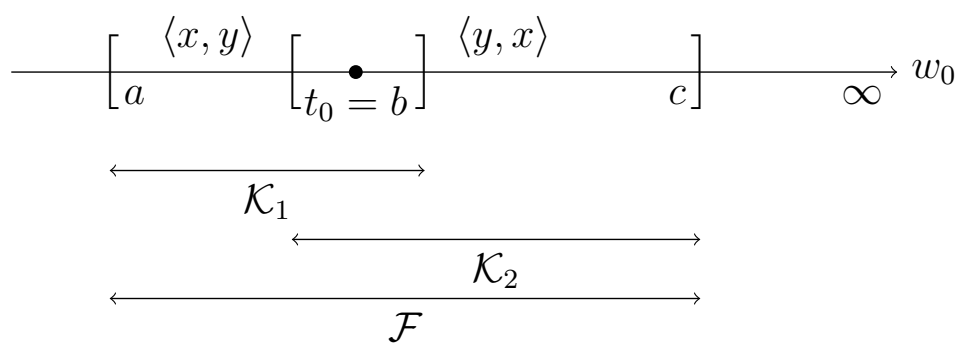

Figure 8: Strong reciprocity in $w_{0}, \mathcal{F}$, with overlapping subintervals

\footnotetext{
${ }^{21}$ We do not discuss here symmetric relations. For insights on this matter, see Gillon, 2007.
} 
Asymmetric predicates cannot be used when strong reciprocity is enacted in every subinterval of $\mathcal{F}$ or across overlapping subintervals in $\mathcal{F}$.

3. In Figure 9, the two pairs $\langle x, y\rangle,\langle y, x\rangle$ (with $x \neq y$ ) stand in the relation $R$ at two different non-overlapping subintervals of $\mathcal{I}$. Note that (34) authorizes pauses between subintervals. Strong reciprocity is cumulatively enacted across subintervals of $\mathcal{F}$ in $w_{0}$. Asymmetric predicates can be used in this case, as illustrated by the sentence in (37). Interval $\mathcal{K}_{1}$ stands for the period at which $x$ succeeds $y$, interval $\mathcal{K}_{2}$ stands for the period at which $y$ succeeds $x$.

(37) The two men succeed each other to the ownership of the shop for taxation purposes.

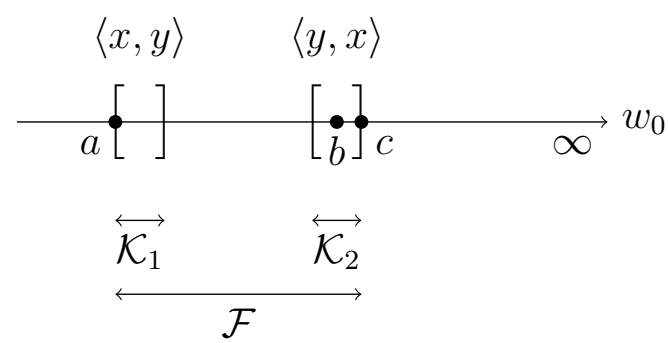

Figure 9: Strong reciprocity in $w_{0}, \mathcal{F}$, with non-overlapping subintervals

Let us now consider the case in which strong reciprocity is not enacted in $w_{0}$ but across non-actual reasonable futures of time $a$.

As illustrated in Figure 10, $x$ stands in the relation $R$ with $y$ in $w_{0}, \mathcal{F}$ (with $\mathcal{F}=\mathcal{K}_{0}$ ), and $y$ stands in relation $R$ with $x$ in a reasonable future of time $a$ (which is not the actual world), at an interval $\mathcal{K}_{1}$. Strong reciprocity is cumulatively enacted across the actual world and non-actual reasonable futures.

The two boxes are stacked on top of each other.

Two boxes remain stacked in one order at $w_{0}, \mathcal{F}$ but they could be stacked in the reverse order in a reasonable future of time $a$, at an interval included in or equal to the reference interval $\mathcal{F}$. Note that reasonable future can branch before or after the utterance time $t_{0}$ (at a time later than time $a$ ).

The following example (39) involves larger groups.

The three boys follow each other.

Let $R=$ follow. As pictured in Figure 11, in $\mathcal{K}_{0}=\mathcal{F}, y$ follows $x$ and is followed by $z$. Different pairs stand in the relation $R$ in non-actual reasonable branches. Intuitively, the order of the boys can change, while the action involving the set of boys continues. 


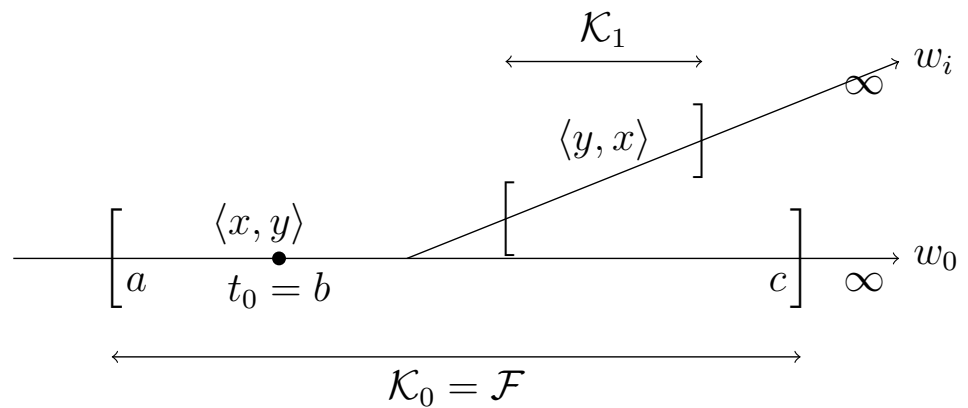

Figure 10: Strong reciprocity cumulatively enacted in the actual world and across branches of the actual world

As pictured, at $\mathcal{K}_{1}$ in $w_{1}, x$ follows $y$ and is followed by $z$; at $\mathcal{K}_{2}$ in $w_{2}, x$ follows $z$ and is followed by $y$; finally, at $\mathcal{K}_{3}$ in $w_{3}, y$ follows $z$ and is followed by $x$.

(For simplicity, in the picture, we use one interval for two pairs of elements standing in the relation $R$ ).

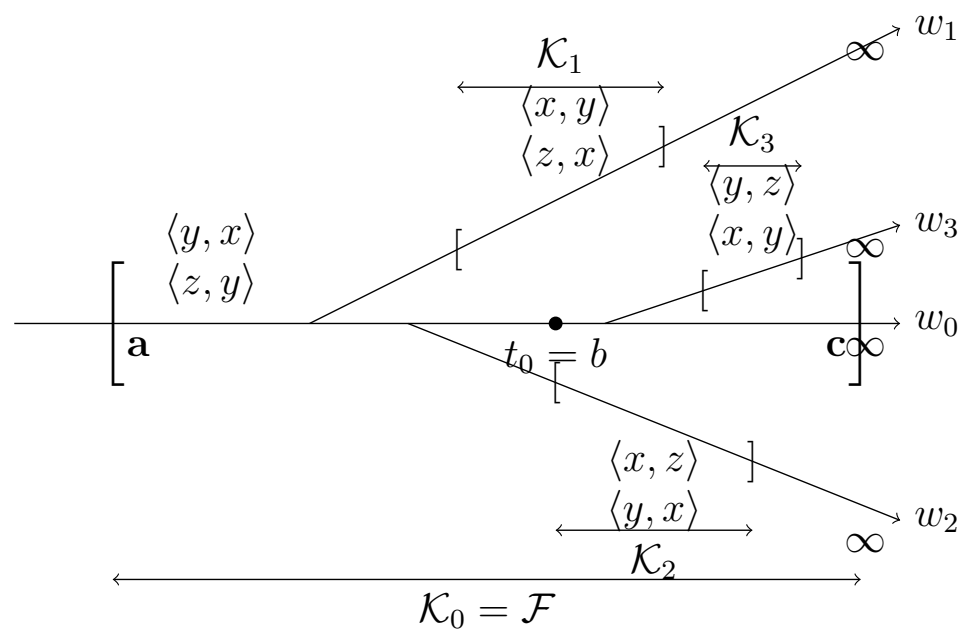

Figure 11: Three boys following each other

\subsection{Strengthening of the rule and past/future asymmetry}

At this point, we need a device for strengthening the rule, which, as it stands, is too weak. ${ }^{22}$ Consider the following example (40).

(40) Scenario: John and Mary were in the middle of a very virulent dispute. John and Mary insulted each other

\footnotetext{
${ }^{22}$ Note that this strategy must be adopted for any theory using ambiguity for the reciprocal or using a variety of rules (although not subscribing to the ambiguity view, see discussion in Beck, 2001 and section 1 here) (e.g., Dalrymple et al. 1998; Beck, 2001; see discussion in Sabato and Winter, 2012 for an alternative approach).
} 
The rule in (34) predicts that (40) is true and acceptable if John insulted Mary in the actual world and if Mary insulted John in one of the reasonable futures determined at the origin of the interval $\mathcal{I}$. This constraint is too weak: (40) requires that Mary insults John and that John insults Mary in the actual world.

In the same spirit, consider the following example (41).

$$
\text { Susan, Mary and Ann combed each other }
$$

Our account predicts that (41) is true if Mary combed Susan and Susan combed Ann and that in a reasonable future in the set determined at the origin of $\mathcal{I}$, Ann combed a different girl in the set of girls. As recently argued in Kerem, Friedmann and Winter (2009), given a set of entities and the predicate comb, it is expected that each of the entities is (actually ${ }^{23}$ ) combed and (actually) combs a different entity in the same set.

Some further constraints must thus be added to (34) to derive the correct predictions. The constraint is the following (42).

In the actual world, the maximum number of pairs of different elements in $A$ allowed by contextual information stand in the relation $R$.

Let us provide some comments, beginning by considering how the 'maximum number of pairs' is determined. This matter has been extensively discussed in Dalrymple et al. (1998) and recently revised in Kerem, Friedmann and Winter (ibid.) and Sabato and Winter (2012). For (41), while Dalrymple et al.'s (ibid.) proposal requires that every entity in the reference set combs and is combed by every other distinct element in the reference set (in this case, each girl combs and is combed by two other girls), Kerem, Friedmann and Winter (ibid.) argue that this requirement is not correct. They explain, for example, that the predicate comb is associated with the concept COMB, which has a typicality preference according to which it is more typical that one combs one person at a time rather than more than one person at a time. The relation denoting one pair of entities (with the first combing the second) better satisfies the typicality conditions associated with the predicate concept than the relation denoting two pairs of entities, with one entity combing two other entities.

For this reason, one could argue that the maximum number of different pairs (of two different elements in $A$ ) standing in the relation $R$ in $w_{0}$ is constrained by typicality preferences associated with the predicate concept.

Note, however, that because we are using intervals, we can obtain strong reciprocity across subintervals, without contravening the typicality preferences associated with the predicate concept. For (41), typicality preferences require that at a given interval, a

\footnotetext{
${ }^{23}$ Kerem, Friendmann and Winter (2009) do not use a modal framework. We employ here the adverb 'actually' to fit the requirement that they propose into our framework.
} 
girl combs at most one other girl and that across subintervals, it is at least the case in the actual world that each girl combs and is combed by a different girl of the same set. Typicality preferences do not tell us what happens across time, and further experimental investigation must elucidate whether speakers tend to maximize the number of entities involved in the relation across subintervals (in the case (41), one would obtain strong reciprocity in the actual world across subintervals). Consequently, the condition requiring maximization of the number of different entities in the reference set that stands in the relation $R$ in the actual world only appeals to contextual information. Future research will determine how lexical and temporal information interact and how (and whether) the rule should be further constrained in view of this interaction.

Contextual information also determines the maximum number of pairs of different elements in $A$ that stand in the relation $R$ in the actual world in a scenario in which a virulent dispute is occurring (40). In this type of scenario, when two participants are involved, it is generally the case that each of the participants insults the others. Provided such contextual information, for (40), maximization of the number of different pairs of two different elements in $A$ that stand in the relation $R$ in the actual world results in Mary also insulting John in the actual world.

Our second comment about (42) pertains to the phrase 'in the actual world'. As noted above, adopting a branching time framework, we have assumed that for any given time $t$, the past of a world at that time is unique, whereas the not-yet-realized-future of that world at that time is one of the options available at $t$. In particular, considering the utterance time $t_{0}$, in this framework, one assumes that the actual world up to and including $t_{0}$ is unique but that there is not yet an actual world after $t_{0}$ and that a variety of options are available. Given this structure of possibilities, one is not entitled to state what the actual-world-to-be is like after $t_{0}$, as this world does not yet exist. Thus, it follows that the constraint in (42) only applies if the actual world is determined, as is the case when the predicate is in the past tense. ${ }^{24}$

Let us illustrate this phenomenon with the following example (43).

\section{Susan, Mary and Ann are combing each other}

Assume that the speaker utters (43) when Susan has combed Mary and Mary has combed Ann but Ann has not yet combed anyone else in the reference set.

Different from what happens with each other past sentences, there is no constraint that can be put on the actual world after $t_{0}$, as there is, in fact, no actual world after $t_{0}$, and one can only reason with reasonable futures.

\footnotetext{
${ }^{24}$ Recall, however, that available options across which strong reciprocity must be realized are determined at the origin of the interval $\mathcal{I}$, which is in the past of $t_{0}$. Once the options are closed, constraint (42) requires that in the world that has become the actual world, the maximum pairs of different elements in $A$ stands in the relation $R$, given certain contextual information.
} 
Clearly, when uttering the sentence, the speaker believes that the not-yet-existing actual world after $t_{0}$ belongs to the reasonable future determined at time $a$ and that Ann will comb Susan in the actual world to come after $t_{0}$. However, this is just a belief, and the constraint in (42) does not apply to each other non-past sentences, as the actual world to come does not yet exist at the utterance time.

Note that even if Ann does not comb Susan in the actual world to come after $t_{0}$, the sentence remains true at $t_{0}$ if, in a reasonable world determined at the origin of the reference interval, Ann combs Susan. More concretely, if the roof under which the girls are combing each other unexpectedly falls down and the girls leave the house before Ann combs Susan, at the time of the utterance (i.e., before the unexpected falling of the roof), the sentence is, nonetheless, true. ${ }^{25}$

To conclude, let us note that for (44), it is also required that John know Mary and that Mary know John.

John and Mary know each other

In this case, the predicate is considered to denote a relation that holds permanently. As we explain in the following section, permanent properties close possibilities, and to satisfy the rule in (34), strong reciprocity must be obtained in the actual world, as it is the case in (44). As we now show, this scenario is possible only with non-asymmetric properties.

Thus, we now turn to the definition of (non-)permanency and (non-)asymmetry in the temporal-modal framework that we have designed.

\section{Definitions: Asymmetry, (non-)decidedness and (non- )permanency}

In this formal section, we define asymmetry and (non-)permanency in the temporal-modal framework that we have designed. We also introduce a new notion of (non-) decidedness for asymmetric relations.

This notion is needed to tease apart cases like (45-a) from cases like (45-b).

a. \#The Eiffel Tower and the Empire State Building are taller than each other

\footnotetext{
${ }^{25}$ We do not discuss here reciprocal each other sentences with the future auxiliary will, which behave like past reciprocal each other sentences: John and Ann will love each other requires that in the future, John loves Ann and Ann loves John. On the assumption that will is a modal universal quantifier that introduces a plan (i.e., that it quantifies worlds that are most similar given the wishes/intentions/beliefs of the speaker or the director of the plan, e.g., Copley, 2002), one understands that the possibilities are metaphysically open but plan-wise closed. Whether this interpretation is true for future sentences and how this information copes with the truth conditions of each other sentences is an issue that remains open.
} 


$$
(=(25))
$$

b. The two tables are stacked on top of each other $(=(17-\mathrm{a}))$

Example (45-a) illustrates a relation that is decided across reasonable futures (fixed at the time of the completion of the Empire State Building). In futures in which no catastrophes occur, and neither the American nor the French government adds or suppresses floors, the Eiffel Tower does not become higher than the Empire State Building.

Example (45-b) illustrates a relation that is non-decided, as across reasonable futures fixed at the time of the stacking of the tables, the order of the tables can change.

The relation holding between two kings in the scenario provided in (46) is also decided. Here, the younger king succeeds the older king who, once succeeded, can no longer succeed his successor.

(46) Scenario: a king cannot succeed his successor.

\# The two kings succeeded each other $(=(24-\mathrm{b}))$

We show that whenever an asymmetric relation is decided, the reciprocal each othersentence is false and unacceptable. As for (45-a), since across the reasonable futures fixed at the time of the completion of the Empire State Building the Eiffel Tower cannot become higher than the Empire State Building, strong reciprocity cannot be enacted and the sentence is ruled out. Similarly, for (46), since across reasonable futures fixed at the time of the succession of the younger king, the older king can no longer succeed his successor, strong reciprocity cannot be enacted and the sentence is ruled out.

In addition, we consider how asymmetry, (non-)permanency and (non-)decideness interact with the truth conditions for reciprocal each other-sentences.

Throughout the remainder of the paper, $t_{0}$ is the utterance time, $w_{0}$ stands for the actual world, and whenever we write $\langle x, y\rangle$ or $\langle y, x\rangle$, we assume that $x \neq y$.

\subsection{Asymmetry}

In our temporal-modal framework, asymmetry is defined as in (47).

\section{Asymmetry}

Let $\mathcal{L}$ be an interval, $A$ a plural set and $W$ a set of worlds. A relation $R$ is asymmetric on $A, \mathcal{L}$ iff

$\forall x, y \in A$ such that $x \neq y, \forall w_{i} \in W, \forall \mathcal{D} \subseteq \mathcal{L}$,

$\left(R\left(\langle x, y\rangle, w_{i}, \mathcal{D}\right)\right) \rightarrow\left((\neg R)\left(\langle y, x\rangle, w_{i}, \mathcal{D}\right)\right)$

Paraphrase: for all $x, y$ in the set $A$ such that $x$ is different from $y$, for all worlds $w_{i}$ in the set $W$, for all intervals $\mathcal{D}$ equal to or included in interval $\mathcal{L}$, if $x$ stands in the relation $R$ with $y$ in $w_{i}$ at $\mathcal{D}$, then $y$ does not stand in the relation $R$ with $x$ in $w_{i}$ at $\mathcal{D}$. 


\section{2 (Non-)decidedness}

The model of branching time represents the past as settled and the future as open. The notion of past settledness is related to that of historical necessity (Aristotle, de Interpretatione IX; Kamp, 1979; Thomason, 1984; Condoravdi, 2002): nothing can change whatever is the case up to and including the present time.

Condoravdi (ibid.) explains that the future can also be represented as settled. In Condoravdi's framework, settledness is a property of common grounds, which are defined as equivalence classes of worlds, fixed at a time $t \in T$. It is not guaranteed that the actual world be part of every common ground. Future settledness is a property of those common grounds that contain specific information to this effect.

In our framework (see section 4.1.2), for any time $t \in T$, we have defined the common ground $c g(t)$ as the set of worlds that are identical to the actual world at least up to and including $t$. (Note that, in our framework, the actual world is part of the common ground). We have then introduced reasonable futures, as the set of worlds determined at $t$ which are part of the common ground, but are such that the rules that hold at $t$ also hold after $t$. (Note that in Condoravdi's framework, there is no notion of reasonability employed). It is possible that the actual-world-to come after $t$ belongs to the set of reasonable worlds.

Here, we define a notion of decidedness that is relativized to reasonable worlds. In particular, we provide a decidedness condition for asymmetric relations in (48). A paraphrase immediately follows.

\section{Decidedness}

Let $\mathcal{L}=[d, \infty$ [ be an arbitrary interval that is unbounded on the right, $A$ a plural set and $R_{\text {asymm }}$ an asymmetric relation. $R_{\text {asymm }}$ is decided on $A, \mathcal{L}, w_{0}$ iff for any two entities $x, y \in A$,

$\left(\exists \mathcal{D} \subseteq \mathcal{L}\left(R_{\text {asymm }}\left(\langle x, y\rangle, w_{0}, \mathcal{D}\right)\right)\right) \rightarrow$ $\left(\forall w_{j} \in \operatorname{ReasFut}(d)\left(\left(\neg R_{\text {asymm }}\right)\left(\langle y, x\rangle, w_{j}, \mathcal{L}\right)\right)\right)$

Paraphrase: if there exists an interval $\mathcal{D}$ equal to or included in interval $\mathcal{L}$ such that $x$ stands in the relation $R_{\text {asymm }}$ with $y$ in $w_{0}$ at $\mathcal{D}$, then for all worlds $w_{j}$ in the set of reasonable futures determined at time $d, y$ does not stand in the relation $R_{\text {asymm }}$ with $x$ in $w_{j}$ at $\mathcal{L}$.

Condition (48) states that an asymmetric relation is decided in $w_{0}$ at the interval $\mathcal{L}=$ $\left[d, \infty\right.$ [ if, whenever the pair $\langle x, y\rangle$ stands in the relation $R_{\text {asymm }}$ at an interval $\mathcal{D} \subseteq \mathcal{L}$ in $w_{0}$, then for all reasonable futures of time $d$, the pair $\langle y, x\rangle$ does not stand in the relation $R_{\text {asymm }}$ in $\mathcal{L}$.

Let us illustrate the decidedness condition. In Figure 12, $\langle x, y\rangle$ stands in the relation $R_{\text {asymm }}$ in a subinterval $\mathcal{D}$ of $\mathcal{L}$ in the actual world, and the pair $\langle y, x\rangle$ does not stand in the relation $R_{\text {asymm }}$ in the interval $\mathcal{L}$, in any reasonable future determined at time $d$. (In 
the pictures, we write $\neg\langle x, y\rangle$, meaning that $x$ does not stand in the relation $R_{\text {asymm }}$ with $y)$.

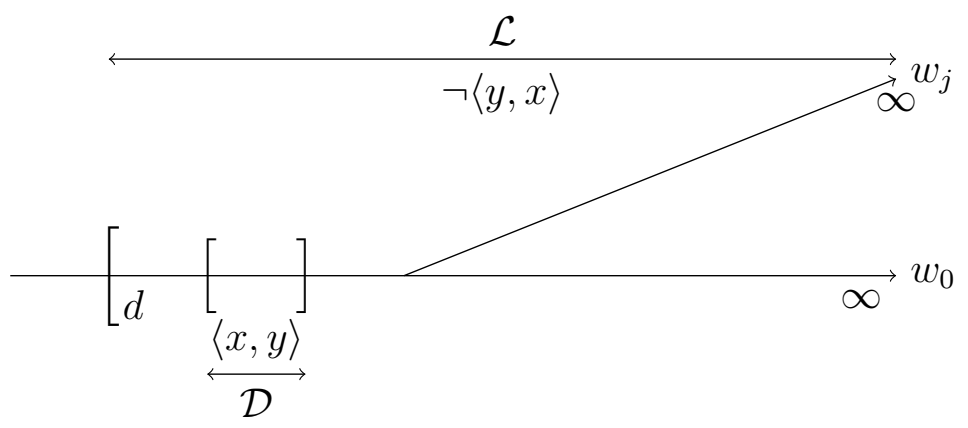

Figure 12: Decidedness, with $R_{\text {asymm }}$ being instantiated at $w_{0}$ at a $\mathcal{D} \subset \mathcal{L}$

In Figure 13, $\langle x, y\rangle$ stands in the relation $R_{\text {asymm }}$ in $\mathcal{L}$ in $w_{0}$, and the pair $\langle y, x\rangle$ does not stand in relation $R_{\text {asymm }}$ in the reference interval $\mathcal{L}$ in any reasonable future determined at $d$.

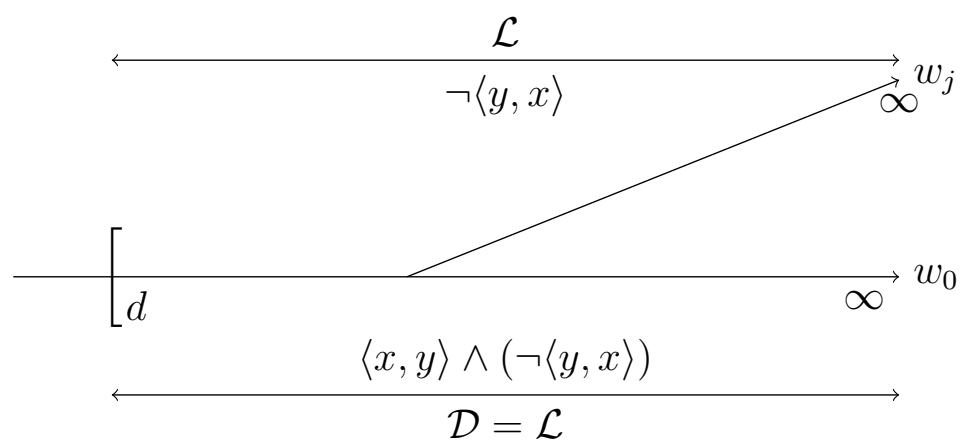

Figure 13: Decidedness, with $R_{\text {asymm }}$ being instantiated at $w_{0}$ at a $\mathcal{D}=\mathcal{L}$

We now make precise the notion of non-decidedness, which is the negation of decidedness. As $\neg(\phi \rightarrow \psi)$ amounts to $\phi \wedge \neg(\psi)$, the negation of (48) reads as (49), which is equivalent to $(50)$.

$$
\begin{aligned}
& \exists x \in A \exists y \in A \\
& \left(\left(\exists \mathcal{D} \subseteq \mathcal{L}\left(R_{\text {asymm }}\left(\langle x, y\rangle, w_{0}, \mathcal{D}\right)\right)\right) \wedge \neg\left(\forall w_{j} \in \operatorname{ReasFut}(d)\left(\left(\neg R_{\text {asymm }}\right)\left(\langle y, x\rangle, w_{j}, \mathcal{L}\right)\right)\right)\right) \\
& \exists x \in A \exists y \in A \\
& \left(\left(\exists \mathcal{D} \subseteq \mathcal{L}\left(R_{\text {asymm }}\left(\langle x, y\rangle, w_{0}, \mathcal{D}\right)\right)\right) \wedge\left(\exists w_{j} \in \operatorname{ReasFut}(d) \neg\left(\left(\neg R_{\text {asymm }}\right)\left(\langle y, x\rangle, w_{j}, \mathcal{L}\right)\right)\right)\right)
\end{aligned}
$$

Now, remark that, for any interval $\mathcal{Z}$ for any world $w \in W, \neg\left(\left(\neg R_{\text {asymm }}\right)(\langle y, x\rangle, w, \mathcal{Z})\right)$ is not equivalent to $\left.R_{\text {asymm }}(\langle y, x\rangle, w, \mathcal{Z})\right)$, rather,

$$
\begin{aligned}
& \neg\left(\left(\neg R_{\text {asymm }}\right)(\langle y, x\rangle, w, \mathcal{Z})\right) \text { iff } \\
& \exists \mathcal{N} \subseteq \mathcal{Z}\left(R_{\text {asymm }}(\langle y, x\rangle, w, \mathcal{N})\right)
\end{aligned}
$$


Using (51), ${ }^{26}$ (52) provides the conditions of non-decidedness.

\section{Non-decidedness}

Let $\mathcal{L}=[d, \infty$ [ be an arbitrary interval that is unbounded on the right, $A$ a plural set and $R_{\text {asymm }}$ an asymmetric relation. $R_{\text {asymm }}$ is non-decided on $A, \mathcal{L}, w_{0}$ iff there exist two entities $x, y \in A$ such that,

$\left(\exists \mathcal{D} \subseteq \mathcal{L}\left(R_{\text {asymm }}\left(\langle x, y\rangle, w_{0}, \mathcal{D}\right)\right)\right) \wedge\left(\exists w_{j} \in \operatorname{ReasFut}(d) \exists \mathcal{B} \subseteq \mathcal{L}\left(R_{\text {asymm }}\left(\langle y, x\rangle, w_{j}, \mathcal{B}\right)\right)\right)$

Paraphrase: there exists an interval $\mathcal{D}$ equal to or included in interval $\mathcal{L}$ such that $x$ stands in the relation $R_{\text {asymm }}$ with $y$ in $w_{0}$ at $\mathcal{D}$ and there exists a world $w_{j}$ in the set of reasonable futures determined at time $d$ such that there exists an interval $\mathcal{B}$ equal to or included in interval $\mathcal{L}$ such that $y$ stands in the relation $R_{\text {asymm }}$ with $x$ in $w_{j}$ at $\mathcal{B}$.

This condition states that there is a pair $\langle x, y\rangle$ that stands in the relation $R_{\text {asymm }}$ in the actual world (at $\mathcal{L}$ or a subinterval of $\mathcal{D}$ of $\mathcal{L}$ ), and there is a reasonable future fixed at time $d$ in which the pair $\langle y, x\rangle$ stands in the relation $R_{\text {asymm }}$, at $\mathcal{L}$ or a subinterval $\mathcal{B}$ of $\mathcal{L}$.

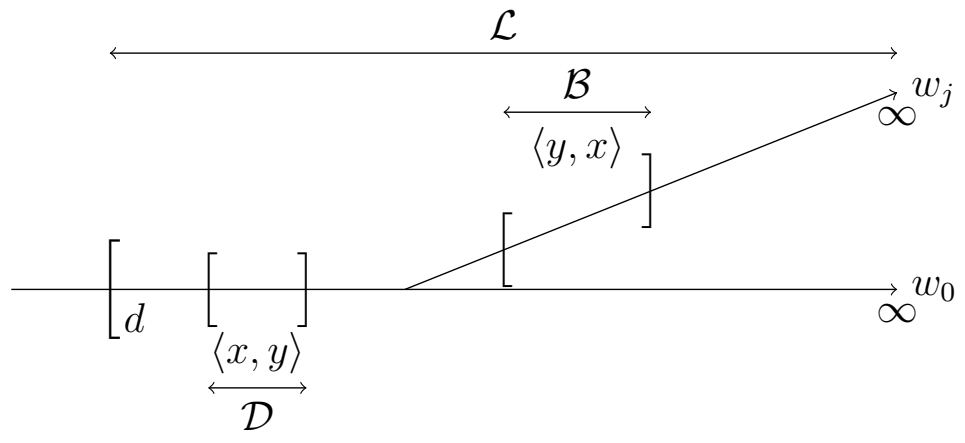

Figure 14: Non-decidedness

For a reciprocal sentence to be true and acceptable, an asymmetric relation should not be decided, but rather non-decided. Before proving this, let us prove that asymmetry of a relation $R_{\text {asymm }}$ is compatible with decidedness, but does not imply it.

An asymmetric relation $R_{\text {asymm }}$ can be decided as well as non-decided on a given interval $\mathcal{L}=[d, \infty[$.

\footnotetext{
${ }^{26}$ Consider the case of two individuals, John and Mary and the 'kiss-on-the cheek' relation. Also, consider a world $w$ and an interval $\mathcal{Z}$ at which John kisses Mary on the cheek and Mary does not kiss John. Letting $x=$ John and $y=$ Mary, we obtain that ( $\neg$ kiss on the cheek) ( $\langle$ Mary,John $\rangle, w, \mathcal{Z})$. To negate this, it is not necessary that Mary kisses John during the whole interval $\mathcal{Z}$. Let $\mathcal{N}$ be a subinterval of interval $\mathcal{Z}$. It is sufficient that Mary kisses John at $\mathcal{N}$. The negation of ( $\neg$ kiss on the cheek) ( $\langle$ Mary,John $\rangle, w, \mathcal{Z}$ ) is thus (kiss on the cheek) $(\langle$ Mary,John $\rangle, w, \mathcal{N})$. Note that here we are treating 'kiss-on-the-cheeck' relation as an atelic one, see discussion in section 3.3 .
} 
Proof. Assume for instance that $w_{0} \in \operatorname{ReasFut}(d)$, and that for some $\mathcal{D} \subseteq \mathcal{L}$, we have $R_{\text {asymm }}\left(\langle x, y\rangle, w_{0}, \mathcal{D}\right)$. So by the very definition of asymmetry we have that $\left(\neg R_{\text {asymm }}\right)\left(\langle y, x\rangle, w_{0}, \mathcal{D}\right)$. Then,

(i)if for all $w_{j} \in \operatorname{ReasFut}(d)$, we also have for all $\mathcal{E} \subseteq \mathcal{L}$ that $\left(\neg R_{\text {asymm }}\right)\left(\langle y, x\rangle, w_{j}, \mathcal{E}\right)$, then $\left.\left(\neg R_{\text {asymm }}\right)\left(\langle y, x\rangle, w_{j}, \mathcal{L}\right)\right)$. In that case, $R_{\text {asymm }}$ is decided on $\mathcal{L}$.

(ii)Suppose now that $R_{\text {asymm }}\left(\langle y, x\rangle, w_{0}, \mathcal{E}\right)$ for some interval $\mathcal{E} \subseteq \mathcal{L}$ such that $\mathcal{D} \cap \mathcal{E}=\emptyset$. Hence, the definition of non-decidedness is checked by taking $w_{j}=w_{0}$ and $\mathcal{B}=\mathcal{E}$ in $(52)$.

We can now prove that, for a reciprocal each other-sentence to be true, an asymmetric relation should be non-decided.

(54) If $E O\left(A, R_{\text {asymm }}\right)$ is true at $w_{0}, t_{0}$, then $R_{\text {asymm }}$ is non-decided on $[a, \infty[$ (where $a$ is defined in (34),(i)).

Proof. Fix $u \in A$. By (34)-(iii), for some $v \neq u$ and some $\mathcal{J} \subseteq[a, b]$ we have $R_{\text {asymm }}\left(\langle u, v\rangle, w_{0}, \mathcal{J}\right)$ or $R_{\text {asymm }}\left(\langle v, u\rangle, w_{0}, \mathcal{J}\right)$. Without loss of generalization, we assume that $R_{\text {asymm }}\left(\langle u, v\rangle, w_{0}, \mathcal{J}\right)$. Then, apply (34)-(iv) to $x=v$ and $y=u$ : for some $c \succeq b$, some $\mathcal{K} \subseteq[a, c]$ and some $w_{j} \in \operatorname{ReasFut}(a)$, we have $\left(R_{\text {asymm }}\left(\langle v, u\rangle, w_{j}, \mathcal{K}\right)\right)$. Thus the definition of non-decidedness on $\mathcal{L}=[a, \infty[$ is verified by taking $\mathcal{D}=\mathcal{J} \subseteq[a, b] \subset[a, \infty[$ and $\mathcal{B}=\mathcal{K} \subseteq[a, c] \subset[a, \infty[$.

\section{3 (Non)-permanency}

We now define permanency within the designed framework.

\section{Permanency}

Let $\mathcal{L}=[d, \infty[$ be an arbitrary interval that is unbounded on the right and $A$ a plural set. A relation $R$ is permanent on $A, \mathcal{L}, w_{0}$ iff, for any two entities $x, y \in A$, $\left(\exists \mathcal{D} \subseteq \mathcal{L}\left(R\left(\langle x, y\rangle, w_{0}, \mathcal{D}\right)\right)\right) \rightarrow\left(\forall w_{j} \in \operatorname{ReasFut}(d)\left(R\left(\langle x, y\rangle, w_{j}, \mathcal{L}\right)\right)\right)$

Paraphrase: if there exists an interval $\mathcal{D}$ equal to or included in interval $\mathcal{L}$ such that $x$ stands in the relation $R$ with $y$ in $w_{0}$ at $\mathcal{D}$, then, for all worlds $w_{j}$ in the set of reasonable futures determined at time $d, x$ stands in the relation $R$ with $y$ in $w_{j}$ at $\mathcal{L}$.

Definition (55) states that, for any two different entities $x, y \in A$, if $x$ stands in relation $R$ with $y$ in the actual world at (a subinterval of) $\mathcal{L}$, it remains in the relation $R$ with $y$ in all reasonable futures determined at time $d$ in $\mathcal{L}$.

We now show that the permanency of an asymmetric relation entails decidedness.

Let $\mathcal{L}=\left[d, \infty\right.$ [ be an interval and $R_{\text {asymm }}$ be an asymmetric relation on $\mathcal{L}$. If $R_{\text {asymm }}$ is permanent in $w_{0}, \mathcal{L}$, then it is decided in $w_{0}, \mathcal{L}$. 


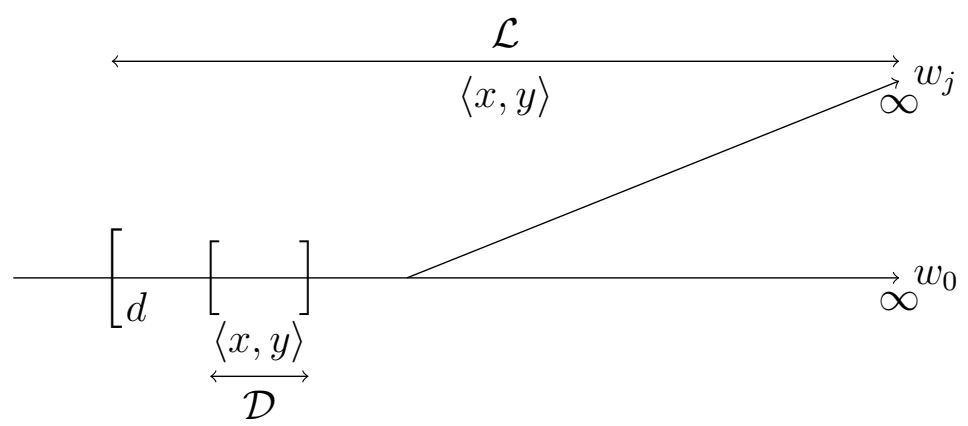

Figure 15: Permanency

PROOF. By asymmetry, we have for all world $w_{j} \in \operatorname{ReasFut}(d)$ that

$$
\left(R_{\text {asymm }}\left(\langle x, y\rangle, w_{j}, \mathcal{L}\right)\right) \rightarrow\left(\left(\neg R_{\text {asymm }}\right)\left(\langle y, x\rangle, w_{j}, \mathcal{L}\right)\right)
$$

So if we assume that $R_{\text {asymm }}$ is permanent in $w_{0}, \mathcal{L}$, then

$$
\begin{aligned}
\left(\exists \mathcal{D} \subseteq \mathcal{L}\left(R_{\text {asymm }}\left(\langle x, y\rangle, w_{0}, \mathcal{D}\right)\right)\right) & \rightarrow\left(\forall w_{j} \in \operatorname{ReasFut}(d)\left(R_{\text {asymm }}\left(\langle x, y\rangle, w_{j}, \mathcal{L}\right)\right)\right) \\
& \rightarrow\left(\forall w_{j} \in \operatorname{ReasFut}(d)\left(\left(\neg R_{\text {asymm }}\right)\left(\langle y, x\rangle, w_{j}, \mathcal{L}\right)\right)\right)
\end{aligned}
$$

i.e. $R_{\text {asymm }}$ is decided in $w_{0}, \mathcal{L}$.

Notice, any reciprocal each other-sentence is predicted false whenever the relation is asymmetric and decided.

(57) Let $\mathcal{L}=\left[d, \infty\left[\right.\right.$ be an interval and $R_{\text {asymm }}$ be an asymmetric and decided relation on $\mathcal{L}$. Then $\operatorname{EO}\left(A, R_{\text {asymm }}\right)$ is false at $w_{0}, t_{0}$.

Proof. The implication (54) amounts for any relation $R$ to

$\left(\neg\left(E O(A, R)\right.\right.$ true at $\left.w_{0}, t_{0}\right) \vee \neg(R$ asymmetric on $\mathcal{L}) \vee \neg(R$ decided on $\left.\mathcal{L})\right)$.

This implies that

$\left(\neg((R\right.$ asymmetric on $\mathcal{L}) \wedge(R$ decided on $\mathcal{L})) \vee \neg\left(E O(A, R)\right.$ true at $\left.\left.w_{0}, t_{0}\right)\right)$,

which amounts in turn to the desired implication.

Therefore, from (56), any each other-sentence is also false whenever the asymmetric relation is permanent.

Using (51), the condition of non-permanency is provided in (58). 


\section{Non-permanency}

Let $\mathcal{L}=[d, \infty[$ be an arbitrary interval that is unbounded on the right and $A$ a plural set. A relation is non-permanent on $A, \mathcal{L}, w_{0}$ iff, there exists two entities $x, y \in A$ such that, $\left(\exists \mathcal{D} \subseteq \mathcal{L}\left(R\langle x, y\rangle, w_{0}, \mathcal{D}\right)\right) \wedge\left(\exists w_{j} \in \operatorname{ReasFut}(d) \exists \mathcal{B} \subseteq \mathcal{L}\left((\neg R)\left(\langle x, y\rangle, w_{j}, \mathcal{B}\right)\right)\right)$ Paraphrase: there exists an interval $\mathcal{D}$ equal to or included in interval $\mathcal{L}$ such that $x$ stands in the relation $R$ with $y$ in $w_{0}$ at $\mathcal{D}$ and there exists a reasonable future $w_{j}$ in the set of reasonable futures determined at time $d$, such that there exists an interval $\mathcal{B}$ equal to or included in interval $\mathcal{L}$ such that $x$ does not stand in the relation $R$ with $y$ in $w_{j}$ at $\mathcal{B}$.

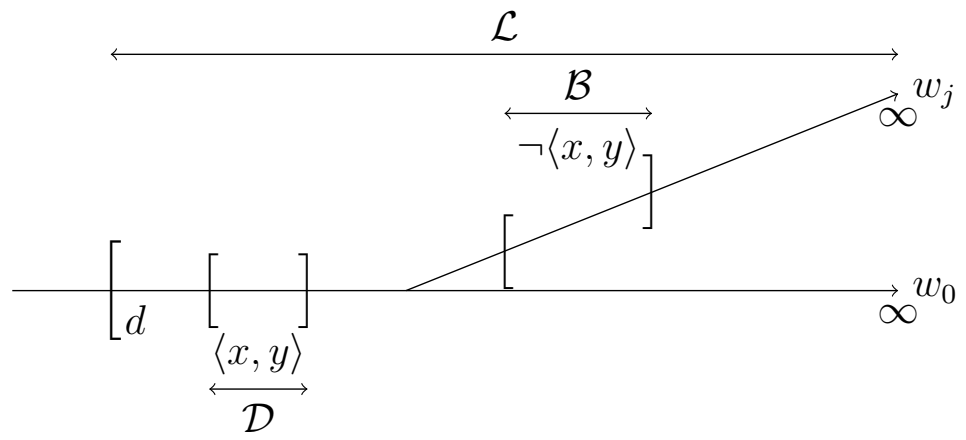

Figure 16: Non-permanency

This condition states that in the actual world, at $\mathcal{D} \subseteq \mathcal{L}$, there is a pair $\langle x, y\rangle$ that stands in the relation $R$, and there is a reasonable future determined at $d$ in which, at an interval $\mathcal{B} \subseteq \mathcal{L}, x$ does not stand in the relation $R$ with $y$.

We now show that a non-permanent asymmetric relation can be decided as well as non-decided.

(59) Let $\mathcal{L}=\left[d, \infty\left[\right.\right.$ be an interval and $R_{\text {asymm }}$ be an asymmetric relation on $\mathcal{L}$. If $R_{\text {asymm }}$ is non permanent on $\mathcal{L}$, then it can be decided as well as non-decided on $\mathcal{L}$.

Proof. Let us assume that for some $\mathcal{D} \subseteq \mathcal{L}, R_{\text {asymm }}\left(\langle x, y\rangle, w_{0}, \mathcal{D}\right)$, that $w_{0} \in$ $\operatorname{ReasFut}(d)$ and that for some interval $\mathcal{B} \subseteq \mathcal{L}$ such that $\mathcal{B} \cap \mathcal{D}=\emptyset$, we have $\left(\neg R_{\text {asymm }}\right)\left(\langle x, y\rangle, w_{0}, \mathcal{B}\right)$. Thus, by taking $w_{j}=w_{0}$ we easily check that $R_{\text {asymm }}$ is not permanent. By asymmetry, $R_{\text {asymm }}\left(\langle x, y\rangle, w_{0}, \mathcal{D}\right)$ entails that $\left(\neg R_{\text {asymm }}\right)\left(\langle y, x\rangle, w_{0}, \mathcal{D}\right)$. Then,

(i)it can very well be that for all $w_{j} \in \operatorname{ReasFut}(d),\left(\neg R_{\text {asymm }}\right)\left(\langle y, x\rangle, w_{j}, \mathcal{L}\right)$ which, for $j=0$, is not incompatible with the fact that $\left(\neg R_{\text {asymm }}\right)\left(\langle x, y\rangle, w_{0}, \mathcal{B}\right)$. This just implies that on $w_{0}$, neither $\langle x, y\rangle$ nor $\langle y, x\rangle$ stand in the relation 
$R_{\text {asymm }}$ on $\mathcal{B}$.

(ii) to the contrary, if we have for instance that $\left(R_{\text {asymm }}\right)\left(\langle y, x\rangle, w_{0}, \mathcal{B}\right)$ (which is not incompatible with $\left(\neg R_{\text {asymm }}\right)\left(\langle y, x\rangle, w_{0}, \mathcal{D}\right)$ since $\left.\mathcal{B} \cap \mathcal{D}=\emptyset\right)$, then the definition of non-decidedness is easily checked by taking $w_{j}=w_{0}$.

As a consequence, a reciprocal each other-sentence can be true when the asymmetric relation is non-permanent.

In summary, we have shown that, when the relation is asymmetric, permanency entails decidedness. Decidedness, however, does not entail permanency. When an asymmetric relation is non-permanent, it can be either decided or non-decided. Whenever an asymmetric relation is decided, the reciprocal each other-sentence is false. Hence, a reciprocal each other-sentence is false when the relation is asymmetric and permanent. For an asymmetric relation, an each other-sentence is true only if the relation is non-decided. So it is true only if the relation is non-permanent.

\section{Predictions}

In this section, we consider the predictions of the account. In section 5.1, we return to the first dataset and show that a reciprocal each other sentence is false and unacceptable whenever the asymmetric relation is decided. We then consider the role of tense and aspect in canceling the inference of persistence associated with the permanent interpretation of the predicates in section 5.2. We review and explain a variety of puzzling contrasts in section 5.3. We return to the question of large groups in section 5.4.

\section{1 (Non-)permanency and (non-)decidedness}

We can now consider the relations between permanency, decidedness and reciprocity returning to the data discussed in section 2 .

\subsubsection{Permanency and decidedness}

Let $d$ be the utterance time and $\mathcal{L}=[d, \infty$ [. Let us consider the following sentence (60), which, as mentioned in section 2, is true and acceptable in a scenario where the head and body have been decapitated and put on top of each other but false and unacceptable when it describes a living being.

(60) (\#)The head and the body are stacked on top of each other.

When sentence (60) is considered to describe a living being and the relation be stacked on top of is considered to hold permanently, reasonable futures are considered in which 
decapitation does not occur.

Let $R=$ be stacked on top of. In the actual world, it holds that there is an interval $\mathcal{D} \subseteq \mathcal{L}$ such that $\left(R\left(\langle\right.\right.$ head,body $\left.\rangle, w_{0}, \mathcal{D}\right)$, and it also holds that in every reasonable future, $w_{j} \in \operatorname{ReasFut}(d),\left(R\left(\langle\right.\right.$ head,body $\left.\rangle, w_{j}, \mathcal{L}\right)$ : the head remains on the body in the actual world and across reasonable branches determined at $d$ (in all subintervals of the reference interval). It is also the case that, in every reasonable future $w_{j}$ determined at time $d$, $(\neg R)\left(\langle\right.$ body,head $\left.\rangle, w_{j}, \mathcal{L}\right)$ (see Figure 13$)$. The relation is thus permanent and decided.

In all sentences in (23), the relation is permanent and decided. These sentences are consequently false and unacceptable.

\subsubsection{Non-permanency and non-decidedness}

In specific scenarios, the relation between the head and the body is not permanent and does not satisfy decidedness. Let $d$ be the utterance time and $\mathcal{L}=[d, \infty[$.

Consider an art museum in which artists create new pieces by manipulating decapitated heads and bodies. In this scenario, there is an interval $\mathcal{D} \in \mathcal{L}$ such that $R\left(\langle\right.$ head,body $\left.\rangle, w_{0}, \mathcal{D}_{1}\right)$, and there is a subinterval $\mathcal{B} \subseteq \mathcal{L}$ in a reasonable future $w_{j}$ determined at $d$ such that $\left(R\left(\langle\right.\right.$ body,head $\left.\left.\rangle, w_{j}, \mathcal{B}\right)\right)$. The choice is given as to whether the decapitated head remains on the body over the entire interval $\mathcal{L}$ in the actual world $w_{0}$ (Figure 10) or it remains on the body over subinterval $\mathcal{D}_{1}$ in $\mathcal{L}$ of the actual world and the body is then stacked on the head in a subsequent subinterval $\mathcal{D}_{2}$ of $\mathcal{L}$ in the actual world (Figure 9). In this case, $\mathcal{B}=\mathcal{D}_{2}$. In this scenario, (60) is correctly predicted true and acceptable.

\subsubsection{Non-permanency and decidedness}

In some cases, the relation is non-permanent and decided. In these cases, the reciprocal each other-sentence is false and unacceptable. Let us reconsider sentence (22) repeated here as (61). Let $d$ be the utterance time and $\mathcal{L}=[d, \infty[$.

(\#)The mushroom pizzas and the pepperoni pizzas are stacked on top of each other.

If pizzas are delivered in stacks of randomly ordered pizzas, (61) is correctly predicted true and acceptable. ${ }^{27}$ If pizzas are delivered in stacks with pepperoni pizzas mandatorily stacked on top of mushroom pizzas, the sentence is correctly predicted false and unac-

\footnotetext{
${ }^{27}$ If there is no rule specifying in what order the pizzas are to be stacked, the sentence is true whether the pizzas are stacked in one or multiple stacks, as any order ('mushrooms on pepperoni' or 'pepperoni on mushrooms') is allowed for each stack. We do not raise the issue here of whether, in this case, quantification is over the reference set $A$ or subsets of the reference set $A$ (Gillon, 1987; Schwarzschild, 1996; Beck, 2001).
} 
ceptable. Assume that the arbitrary interval is the one in which the restaurant's habit of delivering pizzas in stacks with pepperoni pizzas on mushroom pizzas holds.

The relation is non-permanent, as in some reasonable future $w_{j} \in \operatorname{ReasFut}(d)$, there is an interval $\mathcal{B} \subseteq \mathcal{L}$ in which $(\neg R)\left(\langle\right.$ pepperoni,mushroom $\left.\rangle, w_{j}, \mathcal{B}\right)$. It is also decided, though. In the latter scenario, there is an interval $\mathcal{D} \subseteq \mathcal{L}$ in $w_{0}$, such that it holds that $R\left(\langle\right.$ pepperoni,mushroom $\left.\rangle, w_{0}, \mathcal{D}\right)$ and, for all reasonable futures $w_{j} \in \operatorname{ReasFut}(d)$, it holds that $(\neg R)\left(\langle\right.$ mushroom,pepperoni $\left.\rangle, w_{j}, \mathcal{L}\right)$ (recall that reasonable futures are such that habits do not change there, see discussion in section 3.1). The decidedness condition is thus satisfied, and the reciprocal sentence is false and unacceptable.

The relation is also non-permanent and decided in the scenario described in (62).

Scenario: in the seventeenth century, once the older king had been succeeded by the younger one, he could no longer succeed his successor.

\#The two kings succeeded each other. $(=(46))$

Preliminarily, let us note that the past tense is used. In this case, in rule (34)-(i') applies. Here $d$ is the time at which the younger king succeeds the older king. Let $\mathcal{L}=[d, \infty[$ As for (62), given the rules of the country, the relation satisfies decidedness, and strong reciprocity cannot be enacted across intervals in the actual world or across reasonable futures determined at time $d$. Here, the relation satisfies decidedness in the following way. Let $R=$ succeed and the constants $S 1$ and $S 2$ stand, respectively, for the older and younger king. In $w_{0}$, there is an interval $\mathcal{D} \subseteq \mathcal{L}$ such that $R\left(\langle S 2, S 1\rangle, w_{0}, \mathcal{D}\right)$. In every reasonable future $w_{j}$ determined at time $d$ in $\mathcal{L}$, it also holds that $(\neg R)\left(\langle S 1, S 2\rangle, w_{j}, \mathcal{L}\right)$. (see Figure 13). The relation is thus decided. It also holds that there exists a reasonable future $w_{j} \in \operatorname{ReasFut}(d)$ such that there is an interval $\mathcal{B} \subseteq \mathcal{L}$ in which $(\neg R)\left(\langle S 2, S 1\rangle, w_{j}, \mathcal{B}\right)$. The relation is thus non-permanent. However, with the relation decided, the sentence is false and unacceptable.

The account thus predicts that all sentences in (24) are false and unacceptable even if the relation holds non-permanently, because the relation satisfies decidednesss. ${ }^{28}$

\footnotetext{
${ }^{28} \mathrm{An}$ anonymous referee points to us that (i) is not acceptable in a fair coin toss scenario in which it is not decided whether heads or tails comes up, and sees this case as a potential counterexample to our theory. To explain the unacceptability of (i) in this scenario, we construe the minimal pair (i)-(ii). (ii) is acceptable in the scenario provided by the reviewer as predicted by the theory.

(i) \#Heads and tails are on top of each other right now.

(ii) Heads and tails are on top of each other at different tosses.

The question arises of the different contributions of the modifiers at different tosses and right now to sentence (iii) which can be used to describe a coin in general (it is not acceptable in a coin-toss scenario). (iii) Heads and tails are on top of each other.

In (iii) the relation between heads and tails is permanent (note that, even if the relation is asymmetric, (iii) is acceptable differently from the head / body case (\#The head and the body are on top of each other) since heads and tails are in a back-to-back configuration. Heads is on top of tails and Tails is on top of heads are both true, whereas \#the body is on top of head, is false.).

When right now is added, the inference arises that at some point prior to or after the utterance time heads and tails are not on top of each other, with the coin split in two parts. As this is a weird situation,
} 


\subsection{Each other, tense, aspect and the permanent interpretation of the predicates}

Tense choice influences the (un-)availability of the (non-)permanent predicate interpretation and thus the truth/falsity of reciprocal each other sentences. Without even attempting to provide an explanation of how tenses determine the (non-)permanent interpretation (e.g., Condoravdi, 1992; Musan, 1997; Fernald, 2000; Magri, 2009), we acknowledge here a series of facts showing that, when the relation is non-permanent and non-decided and strong reciprocity can be either actually or possibly enacted across reasonable futures determined at the origin of the reference interval, the reciprocal each other-sentence is true and acceptable.

Let us first consider the case of comparatives and, more specifically, the case of the predicate be taller than. Let us start with sentence (63). In contexts that do not consider reasonable futures in which skyscrapers change size, the relation be taller than satisfies permanency (hence decidedness), and the sentence is false and unacceptable.

Scenario: the skyscrapers have reached their final sizes.

\#The skyscrapers are taller than each other.

In other contexts, reasonable futures are considered in which size changes. The sentence becomes true and acceptable, as in (64).

Scenario: a game is played in which participants build skyscrapers, and the one who builds the taller one wins.

The skyscrapers are taller than each other at different stages in their construction.

Here, relation $R=$ be taller than satisfies non-permanency and non-decidedness, as, for any pair $\langle x, y\rangle$ (with $x \neq y$ ), there is a subinterval $\mathcal{D} \subseteq \mathcal{L}$ in the actual world in which $R\left(\langle x, y\rangle, w_{0}, \mathcal{D}\right)$, and there is a world $w_{j} \in \operatorname{ReasFut}(d)$ and a subinterval $\mathcal{B} \subseteq \mathcal{L}$ in which $(\neg R)\left(\langle x, y\rangle w_{j}, \mathcal{B}\right)$ and $R\left(\langle y, x\rangle w_{j}, \mathcal{B}\right)$.

The conditions for obtaining the non-permanent interpretation with the copula be +

the sentence is unacceptable. But why cannot (i) be used in a coin toss scenario like (ii)?

We hypothesize that the possible answer pertains to how lexical and contextual information interact. Interpretation of the reciprocal each other-sentence requires that the relation be non-permanent and nondecided. Right now lexically contributes non-permanency, and non-decidedness is provided contextually. When lexical information is computed, the split-coin scenario is enhanced. The reason why contextual information seems to be unable to rescue the sentence is that right now is of the suitable type, i.e. it provides non-permanency, which is (part of) the expected contribution for the reciprocal sentence to be true and acceptable. This case differs from standard semantic type clashes that are solved by the use of contextual information, as for the omelet at table 4 is fat, where fat requires an argument of type +human and contextual information solves the clash (enabling type coercion).

At different tosses contributes instead non-permanency and non-decidedness at once, enhancing a scenario in which a coin non-permanently stands either with heads up or with tails up and not a splitcoin-scenario. 
adj are complex (see e.g., Maienborn, 2007; Moltmann, to appear).

Let us first consider the case of the past tense, which is simpler than that of the present tense. The use of overt adverbs enables the non-permanent predicate interpretation. As Musan (1997) explains, for (65-a), one infers that Mary's brother is dead and that he was tall his entire life, that is to say, being tall was a permanent property of Mary's brother (Musan, 1997 explains how the lifetime effect is related to the permanent interpretation of the predicate). What matters here is that the permanent predicate interpretation does not arise in (65-b). If one infers that Mary's brother is dead at the time of the utterance, then one also infers that he died at a stage later than his adolescence and that Mary's brother was tall during his adolescence and not later.
a. Mary's brother was tall.
b. Mary's brother was tall during his adolescence.

When the reciprocal is used, the sentence is true and acceptable only when the nonpermanent interpretation of being tall is obtained by the use of overt adverbs.

a. \#My sons were taller than each other.

b. My sons were taller than each other at different stages in their lives.

When the copula is in the present tense, the reciprocal sentence remains false and unacceptable even when overt adverbs are used. ${ }^{29}$

a. \#My sons are taller than each other.

b. \#My sons are taller than each other at different stages in their lives.

However, as already noted for (64), reciprocal each other-sentences are not always false and unacceptable when the copula is in the present tense, notably in fictional contexts (68-a), when rules are expressed (68-b) or in generic sentences (68-c).

a. In this story, the characters are taller than each other at different stages.

b. In this game, the towers are taller than each other at different stages of the game.

c. Siblings are taller than each other at different stages in their lives.

An explanation for the contrast between (67-b) and (68) seems to go beyond a reciprocal theory (e.g., Magri, 2009 for a discussion of the contrast in (69); see also Musan, 1997), as the same truth/falsity patterns arise in non-reciprocal contexts.

\footnotetext{
${ }^{29}$ This and the following observations concern the combination of the copula with adjectives that denote properties that are by default permanent as well as with adjectives that denote non-permanent properties (e.g. '\#The glasses are fuller than each other', Sabato and Winter, 2011). On this matter, see, e.g., Maienborn (ibid.) and Moltmann (ibid.).
} 
a. \#John is sometimes taller than his brother.

b. This character is sometimes taller than this other one.

Furthermore, both (67-a) and (67-b) are false and unacceptable even if I predict (based on some available evidence) that each of my children will be taller than the other one in turn.

These facts seem to be related to the more general observation that the copula in the present tense plus an adjective cannot be used to express plans or forecasts. (70-a) is acceptable only if the state be 1.50 meters tall is coerced into an event 'becoming 1.50 meters tall' (and I have the magic power of deciding what size my son will be next year).

a. $(*)$ My son is 1.50 meters tall next year.

b. My son will be 1.50 meters tall next year.

To express a forecast ${ }^{30}$ with copula plus adjective, one must use the future will (Copley, 2002), both in non-reciprocal (70-b) and reciprocal contexts (71-b). In this case, to obtain the non-permanent interpretation of the predicate be taller than, the use of overt adverbs is preferred (71-b) vs. (71-a).

a. ??My sons will be taller than each other.

b. My sons will be taller than each other at different stages in their lives.

While a study of conditions for obtaining the non-permanent predicate interpretation with the copula plus adjective is a matter that goes beyond the reciprocity question (Condoravdi, 1992, McNally, 1993, Musan, 1997, Maienborn, 2007, Magri, 2009, Moltmann, to appear), these data show that, whenever the conditions are gathered for strong reciprocity to be either actually or possibly enacted over the reference set $A$, the reciprocal each other-sentence is true and acceptable (see (66-b), (68) and (71-b)).

To further illustrate the point, let us reconsider the case of abilitative readings. If (72) is uttered in a context where general abilities of the men are being described (i.e., abilities that are deemed not to change over time, and thus satisfy our definition (55), e.g., Kenny, 1963; Dahl, 1975; Chierchia and McConnell-Ginet, 2000; Menendez-Benito, 2005), the sentence is ruled out. According to this interpretation, strong reciprocity cannot be either actually or possibly enacted across reasonable futures.

(72) Scenario: generic abilities are being described.

\#These men run faster than each other.

In a scenario that describes an ongoing race, the sentences are true and acceptable $((73-\mathrm{a})$ and $(73-\mathrm{b}))$.

\footnotetext{
${ }^{30}$ In contexts which do not include those in (68).
} 
a. These men run faster than each other at each lap.

b. These men are running faster than each other at each lap.

If an ongoing race is described, strong reciprocity can be enacted in the actual world across different subintervals (if each man runs more than one lap, and each time he runs, he runs at a different speed than the previous lap) or reasonable futures (as one can assume that the speed at which each man runs varies across reasonable futures). Speakers prefer (73-b) to (73-a). As we already noted, the progressive does not have a generic interpretation (e.g., Krifka et al. 1995). Moreover, it triggers reasonable futures (e.g., Dowty, 1979; Landman, 1992) and thus easily combines with the reciprocal expression each other. An analysis of how the progressive combines with each other remains outside the scope of this paper.

\subsection{Puzzling contrasts explained}

The puzzling contrasts pertaining to sets of two elements can also now be explained. For all sentences in (74), strong reciprocity cannot be enacted in the scenario mentioned for each case, as required by the truth conditions provided for reciprocal each other-sentences in (34) because the asymmetric relation satisfies decidedness (52).

a. Scenario: one cannot give birth to her/his own mother. \#My mother and I gave birth to each other.

b. Scenario: in the seventeenth century, once the older king had been succeeded by the younger one, he could no longer succeed his successor.

\# The two kings succeeded each other. $(=(62))$

c. Scenario: the head and body of a living being are described. \# The head and the body are stacked on top of each other.

d. Scenario: in a restaurant, the pizzas come in stacks such that pepperoni pizzas are always stacked on top of mushroom pizzas.

\#The mushroom pizzas and pepperoni pizzas are stacked on top of each other.

In the scenario for (74-a), in all reasonable futures fixed at the origin of the reference interval, one cannot give birth to one's own mother; in (74-b), in all reasonable futures fixed at the origin of the reference interval, a king can no longer succeed his successor. As for (74-c), in reasonable futures, the head of a living being cannot be stacked below the body. Finally, in (74-d), as explained above, the sentence cannot go through because the order of the pizzas is never reversed, but they are served with pepperoni pizzas always stacked on top of mushroom pizzas. 
Changing the assumptions such that the asymmetric relation does not satisfy decidedness in reasonable futures fixed at the origin of the reference interval makes the same sentences true and acceptable, as illustrated in (75). In a fictional scenario in which a son can give birth to his own mother, (75-a) becomes true; likewise, (75-b) is true when two kings alternate reigning over the country. As explained above, once a head has been decapitated from its body, (75-c) also becomes true. Finally, (75-d) is true if the restaurant delivers the pizzas randomly stacked, also explained above.

a. Scenario: in a fictional scenario, mothers give birth to their children and die, and the children then give birth to their own mothers.

My mother and I gave birth to each other.

b. Scenario: two kings reign alternately over the country.

The two kings succeeded each other.

c. Scenario: a head and its body have been separated from each other and put back together again in a modern art museum.

The head and the body are stacked on top of each other.

d. Scenario: The pizzas are stacked randomly.

The mushroom pizzas and pepperoni pizzas are stacked on top of each other.

\subsection{Large groups?}

Let us reconsider now the contrast between small and large groups. Recall that previous accounts (e.g., Langendoen, 1978; Dalrymple et al. 1998, Beck, 2001) noted that reciprocal each other-sentences are sometimes unacceptable when the reference set is of cardinality 2 ((76-a) and (78-a)). They also held that when using the reciprocal each other, large groups are preferred ((77-b) and (78-b)). Previous accounts, though, did not explain why large groups do not always rescue the sentence (76-b) and why, when the reference set is of cardinality 2 , the sentence is not always acceptable ((78-a) vs. (77-a)).

(76) Scenario: skyscrapers do not change size.

a. \#The two skyscrapers are taller than each other.

b. \#The skyscrapers are taller than each other.

(77) a. The two boys follow each other.

b. The boys follow each other.

(78) Scenario: once the older king had been succeeded, he could no longer succeed his successor.

a. \# The two kings succeeded each other.

b. The kings succeeded each other. 
The account proposed here divides the data in a different manner and treats large and small groups on a par.

Whether the group is small or large, our account predicts that the sentences are true and acceptable if the relation satisfies non-decidedness (and strong reciprocity is enacted across reasonable possibilities). This is the case for the two sentences in (77).

Whether the group is small or large, our account predicts that the sentences are false and unacceptable when the asymmetric relation satisfies decidedness. In (76), the relation satisfies decidedness, and the sentences are thus predicted false.

The problem raised by the pair in (78) is that the relation satisfies decidedness, yet, contrary to what our account predicts, (78-b) is acceptable (it is instead predicted false and unacceptable).

A corpus study shows that the ban on decidedness is almost exclusively ${ }^{31}$ overwritten in historical and legend reporting contexts.

In (78-b), the relation is non-permanent and decided. The sentence describes a sequence of years/generations that, once passed, can no longer occur and is thus used to convey that time passes irreversibly.

Overwriting decidedness thus seems to be an allowance for the specific purpose of conveying that time has/is passing, and long sequences of years/generations are preferred in this use. To this end, the reference set must be large.

Although these cases are counterexamples to our theory, this restricted use should not hinge on the general reciprocity theory. An explanation of these cases would have to resort to some other pragmatic principles that, at present, remain to be studied. ${ }^{32}$

\footnotetext{
${ }^{31}$ A relation that remains problematic for our account is the containment relation (Sabato and Winter, 2011). A sentence such as 'The Russian puppets are nested into each other' is judged true even if strong reciprocity cannot be enacted (note that, when the set is of cardinality 2, the sentence is false and infelicitous, following the pattern in (78): '\#The two Russian puppets are nested into each other'). As a matter of fact, the relation must be non-permanent, as '\#The Russian puppets are bigger than each other' is judged false. The question we then should address is whether there is a ranking on the constraints, and 'non-permanency + decidedness' is better ranked than 'permanency + decidedness', and whether the reciprocal expression each other is on the way of being used to describe relations that are decided but non-permanent. As Sabato and Winter (ibid.) note, each other is not the preferred reciprocal expression for containment relations. The question must be raised then if the use of each other in such scenarios is new.

In most contexts that describe a containment relation, each other is used when strong reciprocity is possible or even actual, e.g., 'See our hearts contain each other' heylog.com/leanethegirl/36322.

${ }^{32}$ As suggested in the previous footnote, one direction of investigation could consider the interaction between (non-)permanency and (non-)decidedness. One way to describe the phenomenon would be the following: while permanent and decided asymmetric relations are always banned from each otherreciprocal sentences and non-permanent and non-decided asymmetric relations are always admitted in those sentences, historical and legend reporting contexts allow the use of decided and non-permanent asymmetric relations. One would then have to determine whether weakening of the ban against "nonpermanent + decided' asymmetric relations holds specifically for historical contexts (and, in this case, why?), or whether a general tendency to allow 'decided + non-permanent' asymmetric relations is emerging diachronically. If this is the case, a plausible explanation of this phenomenon is that the constraint 'if permanent then decided' is on the way to being reinterpreted as 'permanent if and only if decided'. This would explain that whenever the relation is non-permanent, the reciprocal each other-sentence is true. Whether this reinterpretation is on the way to being carried out can only be stated on the basis of
} 
This conclusion equally leaves the question of atemporal relations unanswered. The two sentences in (79) have the same truth conditions, as they describe an atemporal relation that cannot satisfy strong reciprocity over the reference set. However, (79-a) is acceptable.

a. The numbers follow each other.

b. \#The numbers are greater than each other.

Another potential problem for our theory is represented by (80) (C. Kennedy, p.c.).

The boys are successively taller than each other ${ }^{33}$.

The sentences in (79-a) and (80) seems to share a feature in common, though.

Sentence (80) without the adverb successively is odd (C. Kennedy, p.c.). It can be uttered by someone describing the sizes of a set of boys standing in a line, provided she/he goes from one boy to the other. The sentence describes a 'path' in which the criterion for moving on from one position to the other is to reach boys with greater sizes.

In the same line of thought, we suggest that (79-a) describes a path from one number to the next ${ }^{34}$.

How path semantics relates to semantics of sentences such as (78-b), and more generally to the semantics of each other is a new exciting question that remains unanswered here.

\section{Times and events}

In this section, we compare the coverage of our theory with that ensured by theories that have resorted to temporal (but not modal) considerations. We show, in particular, that theories that have resorted to time can rule out sentences in (23), i.e., those sentences in which the predicate denotes a permanent relation. Accounts uniquely based on time, however, cannot capture cases in (24), in which the predicate denotes a relation that holds non-permanently, but the sentences are nonetheless unacceptable. Our view rules out both of these sets of sentences. In turn, we discuss the theories of Schein (2003) and Beck and von Stechow (2007).

\footnotetext{
diachronic considerations.

${ }^{33}$ With an anonymous reviewer we note that the sentence 'My mother, I and my daughter successively gave birth to each other' also improves with respect to '\#My mother, I and my daughter gave birth to each other'.

${ }^{34}$ The same suggestion applies to the case discussed in footnote 8.
} 


\subsection{Sets of events}

Schein frames the analysis of sentences such as (81-a) in a Parsonian setting (Parsons, 1990) and endorses the assumption that any distributive quantifier is immediately followed by an event argument. The author claims that such sentences describe a point-wise characterization of events of e.g. being stacked on top of, taking one individual at a time, namely, the individual bound by the distributive quantifier. Each individual participant, in his/her/its own event, is added to the set of other events that have already occurred, events of, e.g., stacking. Schein analyzes each other as each the others, where the others is a definite description that is associated with a set event. The truth conditions assigned to (81-a) are in (81-b). The paraphrase is from Schein (ibid.).

a. The plates are stacked on top of each other.

b. $\exists E[$ the $X:$ plates $[X]]$ (stack[E]\& Theme $[E, X] \&$

$[\iota X:$ Theme $[E, X]][$ Each $x: X x]\left[\iota E^{\prime}:\right.$ Overlaps $\left[E^{\prime}, E\right] \&$ Theme $\left.\left[E^{\prime}, x\right]\right]$

$\left[\exists E^{\prime \prime}: t\left(E^{\prime \prime}\right) \leq t\left(E^{\prime}\right)\right]\left[\iota Y\right.$ : Others $[x, Y] \&$ Theme $\left.\left[E^{\prime \prime}, Y\right]\right]$

on-top-of $\left.\left[E^{\prime}, x, Y\right]\right)$ (Schein, 2003:346)

c. The plates stack,

(with) them each stacking

on top of the others' stack.

These truth conditions are intended to capture that each plate $x$ is stacked in its own event $E^{\prime}$ on top of the others $Y$ that are the theme of event $E^{\prime \prime}$, with $E^{\prime \prime}$ preceding or overlapping with $E^{\prime}{ }^{35}$

There are two ways of considering Schein's account: a benevolent or less benevolent one. We adopt the benevolent one and assume that the theory can tease reciprocal sentences with non-permanent relations apart from those in which the relation is permanent. One can assume that only the first class of sentences describe a point-wise characterization of events and are thus predicted true by the account.

In this view, all sentences in (23) are correctly ruled out, as they are not considered to describe a point-wise characterization of events.

Even in this benevolent view, Schein's theory cannot rule out sentences in (24), as these sentences, such as (81-a), describe point-wise event characterizations but are nonetheless false. ${ }^{36}$

\footnotetext{
${ }^{35}$ Schein claims that the first plate is not necessarily stacked on another different plate, as the second order definite description the others should not be taken by itself to assert the existence of those it describes.

${ }^{36}$ There is a less benevolent understanding of Schein's theory. This less benevolent view assumes that, subscribing to a Parsonian view, Schein endorses the claim that all types of relations have an event argument. It would follow that sentences in (23) are predicted true, as they describe a point-wise characterization of overlapping events (e.g., for (23-b), the event in which each man is smart overlaps with the event in which the men less smart than him are smart), an interpretation that is allowed by Schein's truth conditions in (23a).
} 
As a consequence, Schein's theory cannot make sense of the following contrasts. (82-a) describes succession events, much as (82-b) describes following events. (83-a) describes stacking events, much as (83-b) describe procreation events. Only (82-b) and (83-a) are acceptable.

(82) a. Scenario: the older king dies.

\#The two kings succeeded each other.

b. The two boys followed each other.

a. The two boxes are stacked on top of each other.

b. \#My mother and I gave birth to each other.

\subsection{Sequences of events}

Beck and von Stechow (2007) have proposed treating sentences such as (2) with other constructions that describe sequences of events, such as (84-a) and (84-b).
a. She laid book upon book and built a staircase long enough to climb up and look over the wall.
b. My grandmother had on not just one skirt, but four, one over the other.

The authors thus introduce event sequences based on comparisons with the constructions just mentioned. Above each other, for instance, is interpreted as '(one) above the other.'

The paraphrase for $(85-\mathrm{a})$ is $(85-\mathrm{b})$. The truth conditions are informally provided in $(86) \cdot{ }^{37}$

(85) a. The three dogs followed each other into the room.

b. The three dogs came into the room after one another.

(86) For a set event $e, e$ can be divided into a sequence of subevents, and the three dogs can be divided into a sequence of individual dogs such that each dog entered the room in a relevant subevent, and its predecessor entered in the preceding subevent, and each subevent was one of the dogs entering, and the preceding event was one of the predecessors of that dog entering.

As noted by the authors themselves, these truth conditions require that each dog enter after its predecessor. Because the first dog in the sequence does not have a predecessor, sentences of this kind can never be true. To solve the problem, they appeal to pragmatics, which, according to the authors, lets us forget the first dog.

\footnotetext{
${ }^{37}$ See Beck and von Stechow (2007) for formal analysis details.
} 
One advantage of our analysis is that it does not require 'forgetting' the first element of the sequence, as strong reciprocity can be enacted across reasonable futures (Figure 11).

Furthermore, Beck and von Stechow's view encounters the same shortcomings that Schein's theory faces.

On the assumption that only reciprocal sentences with non-permanent relations describe event sequences, Beck and von Stechow can correctly rule out sentences in which the predicate denotes a permanent relation (i.e., (23)) but cannot rule out cases in which the predicates denote a non-permanent relation and the sentence is nonetheless judged false (i.e., (24)).

Beck and von Stechow thus also cannot explain why two member pluralities are used in some but not all cases (see the contrasts in (82) - (83)).

Another concern relates to the role of sentences such as (2) within a unitary view of the meaning of each other. Our analysis has tried to provide such a unitary treatment, making the theory more economic.

\section{Conclusion}

In this paper, we have proposed new truth conditions for reciprocal each other sentences that allow us to explain the constraints on using asymmetric predicates, thus providing new insights into long-standing open questions in the theory of the reciprocal each other. Recalling that only a limited set of predicates denoting an asymmetric relation can be used in reciprocal each other-statements, we have revisited previous descriptions, showing that when the relation is asymmetric it must be non-permanent. We have also shown that this constraint is insufficient, and that, in some cases, the relation is non-permanent and the sentence is nonetheless false.

To explain these facts, we have provided new truth conditions, casting the analysis in a branching-time framework. We have designed two distinct notions for common grounds and reasonable futures, which are both relative to time. Reasonable futures fixed at a time $t$ are those futures in which the laws/habits/behaviors that hold at $t$ continue to hold.

The truth conditions state that a reciprocal each other-sentence is true at the utterance time if this determines an interval such that strong reciprocity can be enacted across reasonable futures fixed at the origin of that interval. Considering reasonable futures determined at the interval's origin allows us to reason with the not-yet-occurred part of the actual world, which follows the utterance time.

We have furthermore explained that strong reciprocity can be enacted across reasonable futures, if the asymmetric relation $R$ does not satisfy decidedness. We have defined (non-)decidedness and have proposed a new definition for (non-)permanency in the de- 
signed modal framework. We have demonstrated that, when the relation is asymmetric, permanency entails decidedness. Consequently, the relation should not hold permanently for the each other-sentence to be true. We have also shown that decidedness does not entail permanency. It follows that, when the relation is non-permanent, it can be decided or non-decided and each other-sentences are false and infelicitous when the relation is non-permanent and decided.

Our account leads us to the following speculation about the reciprocal each other and individuation. The long-standing view about permanency informed us that permanent properties are characteristic of an individual. The requirement that an asymmetric relation be non-permanent amounts to stating that it must not be characteristic of an entity. Consequently, the elements with which a given $x \in A$ actually stands in relation $R$ are not determined by characteristic properties of $x$.

However, as we have observed, the relation can be both non-permanent and decided, and we have provided various scenarios in which contextual knowledge determines whether the asymmetric relation is decided.

In addition to contextual knowledge, in determining whether an asymmetric relation is decided, one might want to consider some other factors: for example, the relative size of the objects (can we say, for a small book and a big dictionary, with the small book on top of the big dictionary, that they are stacked on top of each other?) and physical conditions (can we say for a bottle and table in a space where there is no gravitational force and anything can be on top of anything else that the bottle and table are stacked on top of each other? Can we say it for a bottle on a table, on earth?). To determine the criteria for decidedness of asymmetric relations, future research on reciprocity will have to explore in detail the conceptual representations of objects and their relations. This paper has attempted to provide a formal framework for this future research. This formal framework can also be used for answering new problems that this paper has addressed and left unsolved, pertaining to the interaction between the semantics of reciprocals and of the progressive and to the use of atemporal relations in reciprocal sentences in connection with path semantics.

\section{References}

Aristotle, de Interpretatione. Loeb Classical Library.

Bach, Emmon 1986. The algebra of events. Linguistics and Philosophy, 9: 5-16.

Beck, Sigrid 2001. Reciprocals are definites. Natural Language Semantics 9: 69-138.

Beck, Sigrid and von Stechow, Arnim 2007. Pluractional Adverbials. Journal of Semantics 24: 215-254.

Bennett, Michael and Partee Barbara 1972. Toward the logic of tense and aspect in English. Sys- tem Development Corporation, Santa Monica, California.

Chierchia, Gennaro, 1995. Individual-level Predicates as Inherent Generics. In G. 
Carlson and F. J. Pelletier (eds.), The Generic Book. Chicago: Chicago University Press, $176-223$.

Chierchia, Gennaro and McConnell-Ginet, Sally, 2000. Meaning and Grammar. MIT Press.

Condoravdi, Cleo, 1992. Individual-level Predicates in Conditional Clauses, paper presented at the LSA meeting, Philadelphia, PA.

Condoravdi, Cleo, 2002. Temporal Interpretation of Modals: Modals for the Present and for the Past. In D. Beaver, S. Kaufmann, B. Clark and L. Casillas (eds.), The Construction of Meaning. CSLI Publications, 59-88.

Copley, Bridget 2002. The semantics of the future. PhD MIT.

Dahl, Osten, 1975. On Generics. In E. Keenan (ed.), Formal Semantics of Natural Language, London \& New York: Cambridge University Press, 99-112.

Dalrymple, Mary, Makoto, Kanazawa, Yookyung, Kim, Sam, Mchombo, and Stanley, Peter 1998. Reciprocal expressions and the concept of reciprocity, Linguistics and Philosophy 21: 159-210.

Dotlačil, Jakub and Nilsen, Øystein. 2009. The others Compared to each other Consequences for the Theory of Reciprocity. In T. Friedman and S. Ito (eds.), SALT XVIII, Ithaca, NY: Cornell University.

Dougherty, Ray C.,1968. A transformational grammar of co-ordinate conjoined structures. PhD MIT.

Dowty, David 1979. Word Meaning and Montague Grammar. Dordrecht: Kluwer Academic Publishers.

Fernald, Theodore B., 2000. Predicates and Temporal Arguments, Oxford, New York: Oxford University Press.

Fiengo, Robert and Lasnik, Howard 1973. The logical structure of reciprocal sentences in English, Foundations of Language 9: 447-468.

Gillon, Brendan 1987. The Readings of Plural Noun Phrases in English, Linguistics and Philosophy 10, 199-219.

Gillon, Brendan 2007. On the semantics/pragmatics distinction, Synthese DOI 10.1007/s11229007-9186-5.

Heim, Irene, Lasnik, Howard and May, Robert 1991. Reciprocity and plurality, Linguistic Inquiry 22: 63-101.

Higginbotham, James 1981. Reciprocal Interpretation. Journal of Linguistic Re$\operatorname{search}(1): 97-117$.

Higginbotham, James, and Ramchand, Gillian 1997. The Stage-level / Individual-level Distinction and the Mapping Hypothesis, Oxford University Working Papers in Linguistics, Philology 83 Phonetics 2, 53-83.

Kagan Olga 2011. The Actual World is Abnormal: On the Semantics of the Bylo Construction in Russian. Linguistics and Philosophy 34: 57-84. 
Kamp, Hans 1979. The logic of historical necessity. Unpublished Ms. (non vidi)

Keenan, Edward L. 1987. Unreducible n-ary quantifiers in natural language, in Gardenfors, P. (ed.), Generalized Quantifiers: Linguistic and Logical Approaches. Dordrecht: D.Reidel, 109-150.

Kenny, Anthony, J.P. 1963. Actions, Emotions and Will. New York: Humanities Press.

Kerem, Nir, Friedmann, Naama and Winter, Yoad 2009. Typicality effect and the logic of reciprocity, Proceedings of SALT 19, Ohio State University.

Kratzer, Angelika, 1991. Modality. In A. von Stechow and D. Wunderlich (eds.), Semantics: An International Handbook of Contemporary Research, Berlin: de Gruyter, 639-650.

Krifka, Manfred 1998. The Origins of Telicity. In S. Rothstein (ed.). Events and grammar, Dordrecht: Kluwer Academic Publishers, 197-235.

Krifka, Manfred, Francis J. Pelletier, Greg Carlson, Alice ter Meulen, Godehard Link and Gennaro Chierchia, 1995. Genericity: an Introduction. In G. Carlson and F. J. Pelletier (eds), The Generic Book, Chicago, London: University of Chicago Press, 1-124.

Landman, Fred 1992. The progressive. Natural Language Semantics 1: 1-32.

Langendoen, D. Terence 1978. The logic of reciprocity, Linguistic Inquiry 9: 177-197.

Magri, Giorgio, 2009. A Theory of Individual Level Predicates Based on Blind Mandatory Scalar Implicatures, Natural Language Semantics 17(3), 245-297.

Maienborn, Claudia, 2007. On Davidsonian and Kimian States. In I. Comorowski and K. von Heusinger (eds.), Existence: Semantics and Syntax, Dordrecht: Springer Verlag, 107-130.

McNally, Louise 1993. Adjunct Predicates and the Individual/Stage level distinction. Proceedings of WCCFL 12.

Menendez-Benito, Paula, 2005. The Grammar of Free Choice. PhD Dissertation, University of Massachusetts at Amherst.

Moltmann, Friederike, to appear. On the Distinction between Abstract States, Concrete States, and Tropes. In A. Mari, C. Beyssade and F. Del Prete (eds.), Genericity, Oxford: Oxford University Press.

Musan, Renate 1997. Tense, Predicates and Lifetime Effects. Natural Language Semantics 5: 271-301.

Parsons, Terence 1990. Events in the semantics of English: a Study in Subatomic Semantics. Cambridge, Mass: MIT Press.

Rothstein, Susan 2004. Structuring Events: A Study in the Semantics of Lexical Aspect. Blackwell: Oxford.

Rothstein, Susan 2008. Telicity, Atomicity and the Vendler Classification of Verbs. In S. Rothstein (ed.), Theoretical and Crosslinguistics Approaches to the Semantics of Aspect. Amsterdam: John Benjamins. 
Sabato, Sivan and Winter, Yoad 2005. From Semantic Restrictions to Reciprocal Meanings, Proceedings of FG-MOL 2005.

Sabato, Sivan and Winter, Yoad 2012. Relational Concepts and the Logic of Reciprocity. Ms. University of Utrecht (to appear in Linguistics and Philosophy).

Sauerland, Uli 1998. Plurals, derived predicates and reciprocals, in U. Sauerland and O. Percus (eds.). The interpretative tract. MIT Working Papers in Linguistics 25, 177204.

Schein, Barry 2003. Adverbial, Descriptive Reciprocals, Philosophical Perspectives 17, Language and Philosophical Linguistics, 334-367.

Schwarzschild, Roger 1996. Pluralities. Dordrecht: Kluwer Academic Publishers.

Thomason, Richmond 1984. Combinations of tense and modality, in D. M. Gabbay and F. Guenthner (eds.), Handbook of Philosophical Logic: Extensions of Classical Logic, vol. II, Dordrecht: Reidel, 135-165.

Vlach, Frank, 1981. The semantics of the progressive. In P. Tedeschi and A. Zaenen (eds.). Syntax and Semantics 14: Tense and Aspect. New York: Academic Press.

Vendler, Z. 1967. Linguistics in Philosophy. Ithaca NY: Cornell.

Winter, Yoad 2001. Plural Predication and the Strongest Meaning Hypothesis, Journal of Semantics 18: 333-365.

Winter, Yoad 2009. What neutralizes the positive? CRISSP Colloquium, Brussels. 